\title{
Coupling emitted light and chemical yields from stars: $A$ basic constraint to population synthesis models of galaxies
}

\author{
P. Marigo ${ }^{1,2}$ and L. Girardi ${ }^{2,1}$ \\ 1 Max-Planck-Institut für Astrophysik, Karl-Schwarzschild-Str. 1, 85741 Garching bei München, Germany \\ 2 Dipartimento di Astronomia, Università di Padova, Vicolo dell'Osservatorio 2, 35122 Padova, Italy
}

Received 11 January 2001 / Accepted 20 July 2001

\begin{abstract}
In this paper we emphasize the close connection between the chemical and spectrophotometric evolution of stellar systems: chemical yields from stars correspond to a precise fraction of their emitted light. We translate this concept quantitatively. Starting from simple stellar populations, we derive useful analytical relations to calculate the stellar fuel consumption (emitted light) as a function of basic quantities predicted by stellar models, i.e. the mass of the core and the chemical composition of the envelope. The final formulas explicate the relation between integrated light contribution (total or limited to particular evolutionary phases), chemical yields and stellar remnants. We test their accuracy in the case of low- and intermediate-mass stars, and indicate the way to extend the analysis to massive stars. This formalism provides an easy tool to check the internal consistency between the different stellar inputs adopted in galaxy models: the fuel computed by means of the analytical formulas (corresponding to a given set of chemical yields) should be compared to the exact values given by the luminosity integration along the stellar evolutionary tracks or isochrones (corresponding to a given set of spectrophotometric models). Only if both estimates of the fuel are similar, the stellar inputs can be considered self-consistent in terms of their energetics. This sets an important requirement to galaxy models, also in consideration of the fact that different sources of input stellar data are frequently used to model their spectro-photometric and chemical evolution.
\end{abstract}

Key words. stars: evolution - stars: abundances - galaxies: evolution - galaxies: abundances

\section{Introduction}

Galaxy evolution models represent fundamental tools for the interpretation of a wide variety of observational data, going from star counts in our Galaxy to galaxy counts in deep fields. One of the essential assumptions in these models is that we know how stars convert their nuclear fuel into light and newly-synthesized elements. Then, this knowledge is used to model the light emission from galaxies, as well as the chemical enrichment of the galaxy medium and the baryonic mass locked into stellar remnants. The basic formalism is fully described in the pioneering work by Tinsley (1980).

However, it is also a fact that light and chemical evolution of galaxies have been historically developed into two separate disciplines, that we refer to by the generic names of "spectro-photometric models" (SPM) and "chemical evolution models" (CEM) of galaxies. This separation has probably been the result of the very different basic tools and input data required in these two fields, namely: stellar theoretical isochrones and spectra for SPMs, and stellar

Send offprint requests to: P. Marigo,

e-mail: marigo@pd.astro.it chemical yields for CEMs. Other distinctive features of these fields are that in SPMs star formation rates and age-metallicity relations are usually inputs to the models, whereas in CEMs they are outputs of the calculations.

This picture is nowadays rapidly changing, since the present trend is to model complex stellar aggregates like galaxies accounting simultaneously for all their aspects, mainly spectrophotometric and chemical, and possibly including the dynamical one. Some examples of unified SPMs and CEMs of galaxies can be found in Arimoto \& Yoshii (1986, 1987), Bressan et al. (1994), Einsel et al. (1995), Vazdekis et al. (1996), Chiosi et al. (1998), and Ferreras \& Silk (2000). The simplest way to couple CEM and SPM is quite obvious: one may simply re-direct the output from CEM as input to SPM and, as a matter of fact, this choice has been frequently made in literature. In this way the models naturally succeed in reproducing some observed scaling relations, like for instance the colourmagnitude (mass-metallicity) relation of ellipticals (e.g. Bressan et al. 1994).

In other recent astrophysical problems, coupling CEM and SPM may become a must. For instance, the recent 
indications by Ibata et al. (2000; see also Chabrier 1999) that a significant fraction of the detected massive compact halo objects (MACHO) could consist of white dwarfs, would pose non-trivial problems to present chemospectrophotometric models of galaxies: high rates of white dwarf formation at early epochs would not be compatible with the observed deep galaxy counts (Charlot \& Silk 1996), and with the modest chemical enrichment now observed (Fields et al. 2000). Clearly, these studies point at a link between the light of distant galaxies, and chemical abundances and stellar remnants in the local universe. In this regard, we should also mention the development of more "global" analyses (e.g. Madau \& Pozzetti 2000; Fall 2001), that consider the constraints provided by the cumulative emission of cosmic structures (the optical to far-infrared extragalactic background light), and the average metallicity and stellar mass density of the present universe.

However, the fact that nowadays different aspects of galaxy evolution are being considered simultaneously, does not necessarily imply that the resulting models are selfconsistent as a whole. A potential source of inconsistency could reside in the (quite frequent) use of different sources of stellar data as input to SPM (e.g. luminosities and lifetimes) and CEM (e.g. chemical yields and remnant masses).

In this context, the present paper emphasizes the close relation between the chemical and spectrophotometric evolution of galaxies. We derive the expected relations between the integrated light of a stellar population, the chemical yields of their component stars, and the mass of stellar remnants. These provide important constraints to any model of galaxy evolution, which have so far been either not explicitly checked for, or simply neglected.

We propose such a consistency check may be carried out with the aid of an analytical formalism, based on simple relations involving stellar parameters. The check can be performed as follows. First one derives the fuel (emitted light) directly from the luminosities of the adopted stellar tracks or isochrones (Sect. 2), and then compares it with the fuel analytically derived (Sect. 3), which corresponds to the particular set of chemical yields in use. Of course, the test is successful if the two determinations converge to the same result, as fully discussed in Sect. 4.

\section{Single-burst stellar populations}

Single-burst stellar populations (SSPs) are the building blocks of the composite populations we call galaxies. The integrated stellar light in the pass-band $\lambda$ of an SSP of age $t$ is given by ${ }^{1}$

$L_{\lambda}^{\mathrm{SSP}}(t)=\int_{0}^{\infty} \phi\left(M_{\mathrm{i}}\right) L_{\lambda t}\left(M_{\mathrm{i}}\right) \mathrm{d} M_{\mathrm{i}}$

where $M_{\mathrm{i}}$ corresponds to the initial stellar mass, $L_{\lambda t}\left(M_{\mathrm{i}}\right)$ denotes the luminosity along the isochrone of age $t$,

\footnotetext{
1 Effects such as extinction, gas emission, etc. can be considered a posteriori in the models.
}

and $\phi\left(M_{\mathrm{i}}\right)$ is the initial mass function (IMF). This latter is usually normalized so that the total SSP mass is equal to a known quantity $M_{\mathrm{T}}$, e.g. $\int_{0}^{\infty} M_{\mathrm{i}} \phi\left(M_{\mathrm{i}}\right) \mathrm{d} M_{\mathrm{i}}=$ $M_{\mathrm{T}}$. Integrated magnitudes and colours follow straightforwardly from the quantities $L_{\lambda}^{\mathrm{SSP}}(t)$.

From the above equation, and taking a number of justified approximations (see Girardi \& Bertelli 1998), we can also recover the so-called "fuel consumption theorem" of Renzini \& Buzzoni (1986):

$L_{\mathrm{bol}}^{\mathrm{SSP}}(t)=L_{\mathrm{bol}}^{\mathrm{MS}}(t)+A_{\mathrm{H}} b(t) \sum_{j} F_{j}\left(M_{\mathrm{TO}}\right)$.

In brief, this equation tells us that the integrated bolometric luminosity of a SSP of given age can be expressed as the sum of the integrated luminosity along the main sequence $(\mathrm{MS}), L_{\mathrm{bol}}^{\mathrm{MS}}(t)$, and a quantity that is proportional to the total fuel, $F_{\mathrm{T}}=\sum_{j} F_{j}\left(M_{\mathrm{TO}}\right)$, consumed during all the post-main sequence phases of a star with mass equal to the turn-off mass, $M_{\mathrm{TO}}$. In this equation $b(t)$ is the evolutionary flux, i.e. the number of stars leaving the MS per unit time. The fuel consumption, $F_{j}$, represents the bolometric light emitted by all stars in the post-MS evolutionary stage $j$. However, instead of being expressed in energy units, this light is expressed in units of "equivalent-mass of hydrogen burnt" (e.g. in $\left.M_{\odot}\right)$. This energy-to-mass conversion is properly taken into account by the constant $A_{\mathrm{H}}$ in Eq. (2), which expresses the efficiency (i.e. energy released per unit mass) of H-burning reactions. In this work we adopt $A_{\mathrm{H}}=9.75 \times 10^{10} L_{\odot} \mathrm{yr} M_{\odot}{ }^{-1}$, which is derived from the net $Q$-value of $\mathrm{H}$-burning reactions via the $\mathrm{CNO}$ cycle.

Since the burning of a given mass of helium provides only one tenth of the energy that is provided by the nuclear consumption of the same mass of hydrogen (i.e. $\left.A_{\mathrm{He}} \sim 0.1 A_{\mathrm{H}}\right), F_{j}\left(M_{\mathrm{TO}}\right)$ is then given by

$F_{j}\left(M_{\mathrm{TO}}\right) \simeq \Delta M_{\mathrm{H} j}+0.1 \Delta M_{\mathrm{He} j}$,

where $\Delta M_{\mathrm{H} j}$ and $\Delta M_{\mathrm{He} j}$ are the masses of $\mathrm{H}$ and $\mathrm{He}$, respectively, nuclearly burnt during the $j$ th post-main sequence evolutionary phase of the star of initial mass $M_{\mathrm{TO}}$.

From Eqs. (1) and (2) above, it is also clear that

$F_{j}\left(M_{\mathrm{TO}}\right)=\frac{1}{A_{\mathrm{H}} b(t)} \int_{j} \phi\left(M_{\mathrm{i}}\right) L_{t}\left(M_{\mathrm{i}}\right) \mathrm{d} M_{\mathrm{i}}$,

where the integration is limited to the section of the isochrone with age $t$ that corresponds to the $j$ thevolutionary stage only.

On the other hand, if we are dealing with stellar evolutionary tracks instead of isochrones, the fuel of a star with initial mass $M_{\mathrm{i}}$ can be calculated as the time-integral of the luminosity over the duration of that phase, divided by the constant $A_{\mathrm{H}}$ :

$F_{j}\left(M_{\mathrm{i}}\right)=\frac{1}{A_{\mathrm{H}}} \int_{j} L_{M_{\mathrm{i}}}(t) \mathrm{d} t$.

In the previous equations, $F_{j}$ is expressed in $M_{\odot}, L$ is given in $L_{\odot}$, and the independent variable $t$ is expressed in yr. 
From the aforementioned definitions it is immediately clear that the $F_{j}$ are important quantities, because they relate basic properties of stellar structures (Eq. (3)) to the integrated light of SSPs (Eq. (4)). Examples of the practical application of the above concepts can be found in e.g. Greggio \& Renzini (1990) and Renzini (1998). It is just the stellar fuel consumption and its main features the main subject of the analysis we will develop in the following.

\section{Computing the fuel consumption}

Let us now concentrate on the problem of determining, in a generalised fashion, the quantities $\Delta M_{\mathrm{H} j}$ and $\Delta M_{\mathrm{He} j}$, or equivalently $F_{j}$, corresponding to a stellar model of given initial mass $M_{\mathrm{i}}$.

We can devise two methods respectively based on

1. "exact" numerical integration along the track;

2. analytical approximations.

With the first approach the fuel consumed during the $j$ th-evolutionary phase can be calculated from the stellar track by simply using Eq. (5), or, alternatively, by integrating along the corresponding section of the isochrone with $M_{\mathrm{TO}}=M_{\mathrm{i}}$ as in Eq. (4).

The second method relies on simple approximate relations, involving essentially the size of the fuel-exhausted core, and the increase/decrease in the mean masscoordinate of the overlying burning shell. In the context of this approach, we will present here an analytical formalism to derive the fuel, developed in detail for low- and intermediate-mass stars, and briefly sketched for massive stars. The relations are of easy practical application as they contain quantities that can be extracted from available stellar models.

If the first approach is to be preferred in terms of accuracy - as it straightforwardly follows from the definition of fuel consumption itself (see Sect. 2) -, the second approach has the merit of expliciting intimate inter-dependences between (apparently) different properties of stellar populations (i.e. light emission and chemical enrichment). The accuracy of the formalism will be tested by comparison to the results obtained with the "exact" method.

\subsection{Basic considerations}

Two fundamental ingredients in the calculation of the fuel are the mass-coordinates of the $\mathrm{H}$ - and He-exhausted cores. This is already suggested by Eq. (3), that expresses the fuel in terms of two contributions $-\Delta M_{\mathrm{H}}$ and $\Delta M_{\mathrm{He}}$ - which are obviously related to the growth of the $\mathrm{H}$ - and He-exhausted cores, respectively. In this sense we may say that, as the star evolves, the He and CO cores keep track of the fuel being consumed, by accreting the ashes of the nuclear burnings occurring above them. We may also advance that, in general, the derivation of the fuel will involve the difference between the core masses at some "initial" and "final" stages of the phase under consideration.
From Eq. (3), we can also derive some general rules that are valid for any increase of the core mass $\Delta M_{\mathrm{c}}$. If we are considering a $\mathrm{H}$-exhausted core that has grown in mass by simply transforming $\mathrm{H}$ into ${ }^{4} \mathrm{He}$, the corresponding fuel is increased by $\Delta F_{j}=X \Delta M_{\mathrm{c}}$, where $X$ is the original abundance of $\mathrm{H}$ (in mass fraction) in the material nuclearly burnt. If instead, we are dealing with a Heexhausted core that accretes the products of He-burning (mainly carbon and oxygen via the $\alpha$-capture reactions), we would have $\Delta F_{j}=0.1 \Delta M_{\mathrm{c}}$. Finally, if during the same evolutionary stage, a core has grown in mass through the successive conversions of $\mathrm{H}$ into $\mathrm{He}$ and then $\mathrm{He}$ into $\mathrm{CO}$, we would have $\Delta F_{j}=(X+0.1) \Delta M_{\mathrm{c}}$.

However, besides these general and quite simple considerations we should add others which are equally important, though (probably) somewhat less intuitive. We remark, in fact, that the knowledge of the $M_{\mathrm{c}}$ is not sufficient to estimate the fuel, since part of the burnt material initially deposited in the core may be reduced by dredgeup events. The net effect is that products of nuclear burnings previously occurred in situ in core regions (burning shells included) are carried away to the outermost layers (dredge-up), and a fraction of them can also be irreversibly lost by the star (mass loss). Then, we can already expect that this bit of information subtracted from the core is intimately related to the stellar yields, as we will better explicit in Sect. 3.2.

To allow an easier understanding of the formalism developed in this study let us recall now a few relevant aspects of dredge-up and mass-loss processes characterising the evolution of low- and intermediate-mass stars.

The dredge-up episodes - caused by the penetration of the convective envelope into regions previously affected by nucleosynthetic processes - typically occur in red-giant stages (i.e. RGB and AGB) between major phases of core nuclear burning, possibly producing two effects:

- changes in the chemical composition of the envelope; and

- inward shift of (either one or both) the hydrogenhelium and helium-CO discontinuities (or chemical profiles).

Evolutionary stellar calculations (e.g. Girardi et al. 2000; Marigo et al. 1999) predict that the 1st dredge-up occurs at the base of the RGB in low- and intermediate-mass stars of any mass, the 2nd dredge-up takes place only in stars with initial mass $M_{\mathrm{i}} \gtrsim 3-4 M_{\odot}$ at the beginning of the Early-AGB (E-AGB), and the 3rd dredge-up ${ }^{2}$ may occur at thermal pulses during the TP-AGB phase.

The 1st and 2nd dredge-up bring up to the surface material which has undergone H-burning, with a consequent net enrichment of ${ }^{4} \mathrm{He}$ in the envelope. The third dredge-up can contribute to increase the surface ${ }^{4} \mathrm{He}$ abundance as well, at the same time injecting into the envelope

\footnotetext{
${ }^{2}$ This term comprehends all recurrent dredge-up events during the TP-AGB phase.
} 


\section{Low-mass star}

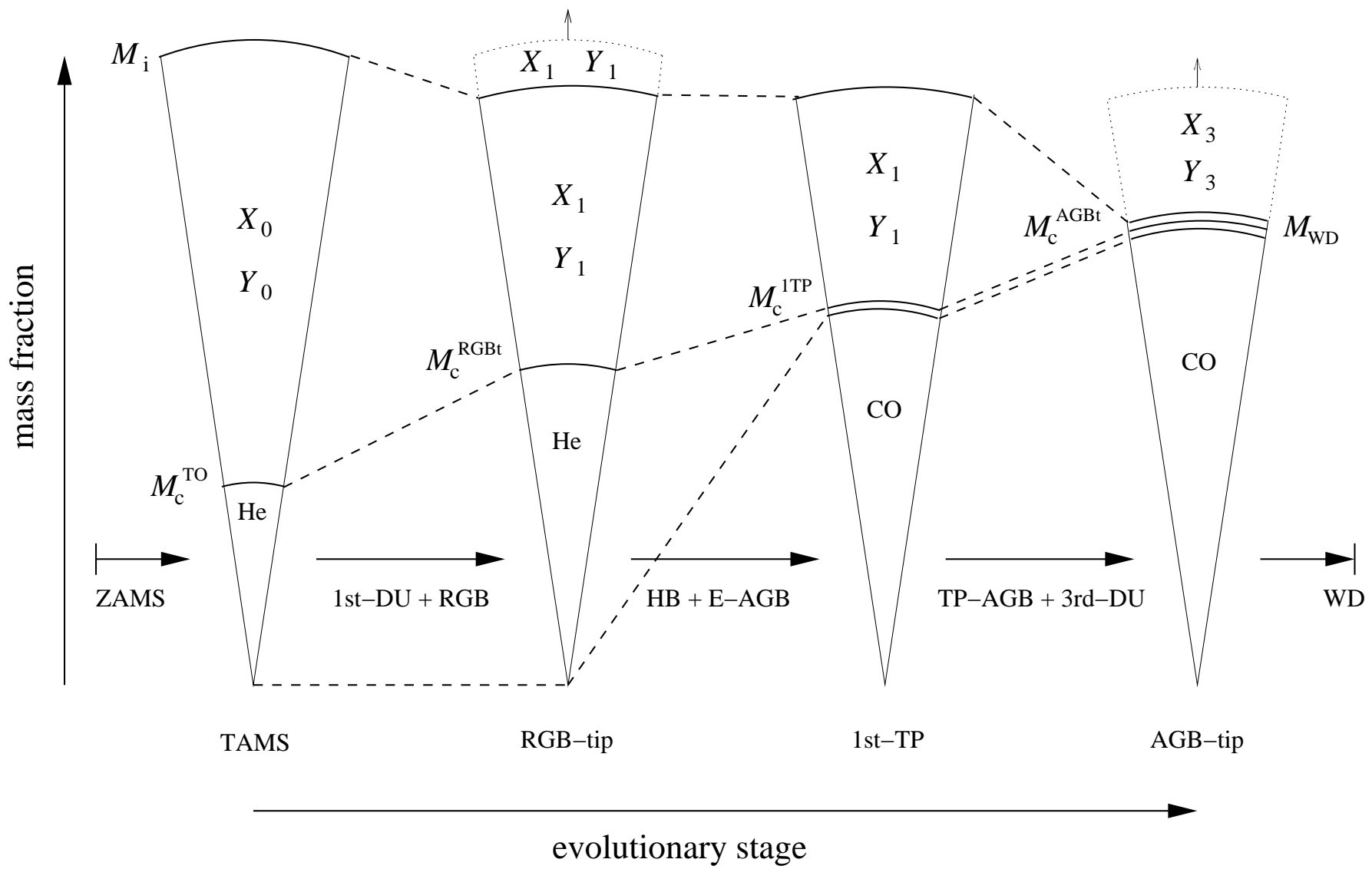

Fig. 1. Schematic representation of the structure of a low-mass stars at several evolutionary stages, namely (from left to right): at the main sequence termination (TAMS), at the RGB tip, at the onset of thermal pulses on the AGB (1st-TP), and at the last thermal pulse (AGB-tip). These phases comprehend the whole post-main sequence nuclear life of these stars. The bold solid lines (connected by dashed lines between stages), delimit the following boundaries (from top to bottom) between: the stellar surface and the mass lost by stellar winds, the H-rich envelope and the (H-exhausted) He core, and the He and CO cores. The chemical composition $(X, Y)$ of the H-rich envelope is modified by the dredge-up (DU) episodes as marked. During the RGB and AGB phases, mass-loss significantly reduces the stellar mass (regions delimited by dots). See text for a complete discussion.

products synthesized at He-shell flashes, mainly ${ }^{12} \mathrm{C}$ and ${ }^{16} \mathrm{O}$ of primary synthesis.

In addition to the aforementioned dredge-up episodes, the surface chemical composition of TP-AGB stars with $M_{\mathrm{i}} \gtrsim 3.5 M_{\odot}$ can be altered by the occurrence of hydrogen-burning via the CNO-cycle at the base of their convective envelopes (hot-bottom burning; HBB). This latter process also increases the surface abundance of ${ }^{4} \mathrm{He}$, and that of ${ }^{14} \mathrm{~N}$ at the expenses of the newly dredged-up ${ }^{12} \mathrm{C}$ (and possibly ${ }^{16} \mathrm{O}$ ).

Another crucial process characterising the evolution of low- and intermediate-mass stars is mass loss via stellar winds, both during the RGB (of low-mass stars) and AGB phases. From both theoretical and observational arguments we get indications that during the final stages of the AGB evolution of these stars, mass loss is able to almost completely strip off their mantles. The final result is a white dwarf consisting almost completely of a $\mathrm{C}-\mathrm{O}$ core, and with very thin (in mass) superficial layers of $\mathrm{H}$ and $\mathrm{He}$
It is just the combination of surface composition changes (due e.g. to dredge-up events) and mass loss that determines the contribution of these stars to the chemical enrichment of the interstellar medium (ISM). Such contribution is commonly quantified through the stellar yields, defined as the amount of newly synthesized elements ejected into the ISM.

\subsection{Analytical derivation}

In order to guide our discussion, Figs. 1 and 2 schematically present the basic structure of low- and intermediatemass stars at some key-evolutionary stages. In our scheme, each star starts as a chemically homogeneous zero-age main sequence (ZAMS) configuration, and ends up as a $\mathrm{CO}$ white dwarf with virtually no $\mathrm{H}$ - or He-rich envelopes. Thus, the evolutionary stages depicted comprehend its whole post-MS nuclear evolution. 


\section{Intermediate-mass star}

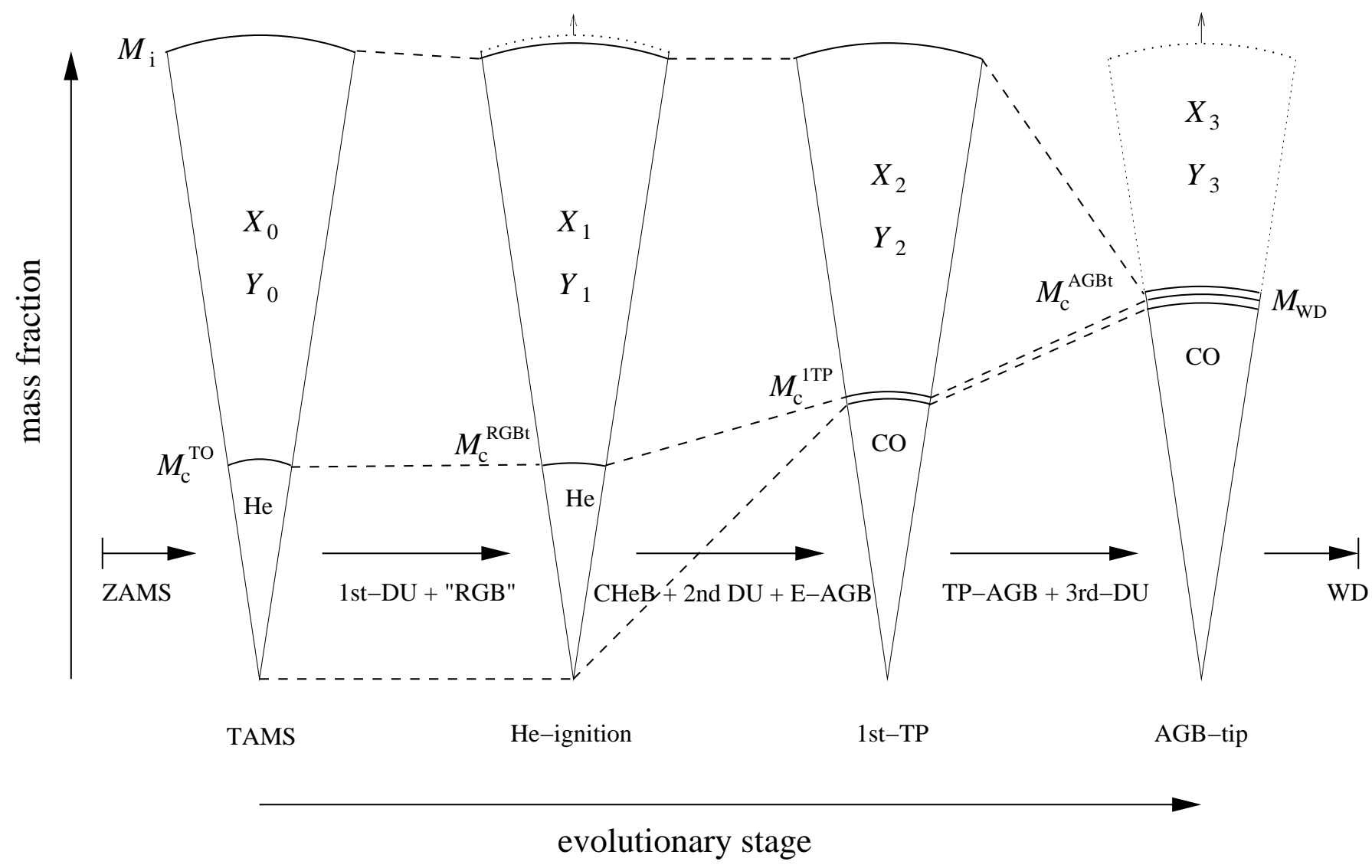

Fig. 2. The same as Fig. 1 but for an intermediate-mass star. In this case, the RGB phase is almost absent and with negligible mass loss.

Each stage is identified by the corresponding mass of the H-exhausted core $M_{\mathrm{c}}$, namely:

- the termination of the main sequence (TAMS). This is equivalent to the turn-off (TO) point of the corresponding isochrone (or SSP) with $M_{\mathrm{c}}^{\mathrm{TO}}$;

- the tip of the RGB (or the stage of central He ignition) with $M_{\mathrm{c}}^{\mathrm{RGBt}}$;

- the first thermal pulse on the AGB with $M_{\mathrm{c}}^{1 \mathrm{TP}}$; and

- the tip of the AGB with $M_{\mathrm{c}}^{\mathrm{AGBt}}$ (notice that $M_{\mathrm{c}}^{\mathrm{AGBt}}$ coincides with the remnant mass $M_{\mathrm{WD}}$ ).

Moreover, it is worth noticing that during the stages under consideration not only the mass-coordinate of the core(s), but also its chemical composition (either a He-core, or a $\mathrm{CO}$-core surrounded by a very thin He-rich layer) can be easily singled out in stellar models. This can be appreciated looking at the lines depicted in Figs. 1 and 2 that schematically describe the evolution of the $\mathrm{H}-\mathrm{He}$ and $\mathrm{He}-$ CO discontinuities.

Outside the $\mathrm{H}$-exhausted core, there is an envelope whose mass is reduced by both the core growth and mass loss, but can be temporarily increased (even if the former decrease is by far dominant) by dredge-up events. These latter events also change the envelope chemical composition. We denote by $X_{j}, Y_{j}$ - with $j=0,1,2,3$ - the envelope fractional abundances (by mass) of hydrogen and helium respectively, where the superscripts indicate the original abundances if $j=0$, and those after the $j$ thdredge-up event if $j>0$.

In general, with the term "core mass" we denote the mass-coordinate of a specified chemical discontinuity (e.g. the mass of the $\mathrm{H}$-exhausted core corresponds to the $\mathrm{H}-\mathrm{He}$ discontinuity). This definition, however, cannot always be applied. For instance, stellar models at the TAMS do not show any sharp H-He discontinuity, but rather a chemical profile - left by either the radiative core or the recession of the convective core-, so that $M_{\mathrm{c}}^{\mathrm{TO}}$ does not correspond to any well-defined mass-coordinate inside the star. Anyhow, we can find a physically equivalent definition for $M_{\mathrm{c}}^{\mathrm{TO}}$, that is

$M_{\mathrm{c}}^{\mathrm{TO}}=\frac{1}{X_{0}} \int_{0}^{M_{\mathrm{i}}}\left[X_{0}-X\left(M_{\mathrm{r}}\right)\right] \mathrm{d} M_{\mathrm{r}}$,

where $X\left(M_{\mathrm{r}}\right)$ is the $\mathrm{H}$ abundance at the mass-coordinate $M_{\mathrm{r}}$. In practice, $M_{\mathrm{c}}^{\mathrm{TO}}$ corresponds to the mean value of $M_{\mathrm{r}}$ across the chemical profile, weighted by the difference between the initial and local $\mathrm{H}$ abundance. We can also notice that the total mass of $\mathrm{H}$ burnt during the MS is equal to $X_{0} M_{\mathrm{c}}^{\mathrm{TO}}$. Moreover, Eq. (5) offers an alternative way to compute $M_{\mathrm{c}}^{\mathrm{TO}}$ from evolutionary tracks:

$M_{\mathrm{c}}^{\mathrm{TO}}=\frac{1}{X_{0} A_{\mathrm{H}}} \int_{\mathrm{MS}} L_{M_{\mathrm{i}}}(t) \mathrm{d} t$. 
At this point we have defined (and illustrated in Figs. 1 and 2) all the ingredients necessary to estimate the nuclear fuel consumed in different evolutionary stages of low- and intermediate-mass stars.

\subsubsection{The total fuel}

Hereinafter we will briefly refer to total fuel as that consumed during the entire post-main sequence evolution. This can be evaluated with

$F_{\mathrm{T}} \simeq\left(X_{0}+0.1\right) M_{\mathrm{c}}^{\mathrm{AGBt}}-X_{0} M_{\mathrm{c}}^{\mathrm{TO}}+F_{y}^{\mathrm{He}}+F_{y}^{\mathrm{CO}}$,

where $\left(X_{0}+0.1\right) M_{\mathrm{c}}^{\mathrm{AGBt}}-X_{0} M_{\mathrm{c}}^{\mathrm{TO}}$ represents the fuel required to convert a He-core of mass $M_{\mathrm{c}}^{\mathrm{TO}}$ into a CO-core of mass $M_{\mathrm{c}}^{\mathrm{AGBt}}$ (see Figs. 1 and 2); $F_{y}^{\mathrm{He}}$ and $F_{y}^{\mathrm{CO}}$ represent the contributions to the fuel related to the stellar yields of ${ }^{4} \mathrm{He}$, and ${ }^{12} \mathrm{C}+{ }^{16} \mathrm{O}$ elements (produced by He-burning reactions), respectively. In this paper, $M_{y}(i)^{3}$ denotes the stellar yield of element $i$ (in mass units).

The term referred to ${ }^{4} \mathrm{He}$ is, by definition, simply

$F_{y}^{\mathrm{He}}=M_{y}(\mathrm{He})$,

with

$M_{y}(\mathrm{He})=\int_{0}^{\tau^{*}}\left[Y(t)-Y_{0}\right] \frac{\mathrm{d} M}{\mathrm{~d} t} \mathrm{~d} t$

where the integral is carried out over the entire stellar lifetime $\tau^{*} ; Y(t)$ and $\mathrm{d} M / \mathrm{d} t$ are the current (at time $t$ ) surface ${ }^{4} \mathrm{He}$ abundance and mass-loss rate, respectively.

The newly synthesised $\mathrm{CO}$ nuclei from He-burning reactions are clearly of primary origin. This applies, for instance, to ${ }^{12} \mathrm{C}$ and ${ }^{16} \mathrm{O}$ nuclei brought up to the surface by the third dredge-up, as they are synthesised at Heshell flashes during the TP-AGB evolution. Denoting with $M_{y}^{\mathrm{P}}(\mathrm{CO})$ the corresponding primary yield (see Eq. (23) below), we can use the approximation:

$$
\begin{aligned}
F_{y}^{\mathrm{CO}} & =1.1\left(1-Y^{\prime}\right) M_{y}^{\mathrm{P}}(\mathrm{CO})+0.1 Y^{\prime} M_{y}^{\mathrm{P}}(\mathrm{CO}) \\
& =\left(1.1-Y^{\prime}\right) M_{y}^{\mathrm{P}}(\mathrm{CO})
\end{aligned}
$$

where

$Y^{\prime}=\frac{Y_{1,2}}{X_{1,2}+Y_{1,2}}$.

This latter quantity involves $\mathrm{H}$ and ${ }^{4} \mathrm{He}$ abundances at the onset of the TP-AGB phase, i.e. after the 2nd dredge-up or, if this latter does not occur as in lower mass stars, after the 1st dredge-up. Equation (12) accounts for the fact that a fraction $Y^{\prime}$ of $M_{y}^{\mathrm{P}}(\mathrm{CO})$ derives directly from nuclear burning of original helium, whereas the complementary fraction $1-Y^{\prime}$ is synthesized starting from original hydrogen through the sequence of both $\mathrm{H}$ - and He-burning.

It is worth remarking that all the quantities that enter in the evaluation of Eq. (8) - i.e. the core masses $M_{\mathrm{c}}^{\mathrm{TO}}$, the

\footnotetext{
${ }^{3}$ For all the definitions related to yields, we refer to the classical work by Tinsley (1980), and to the recent calculations presented by Marigo (2001).
}

remnant mass $M_{\mathrm{c}}^{\mathrm{AGBt}}=M_{\mathrm{WD}}$, the chemical abundances after dredge-up episodes, $X_{j}$ and $Y_{j}$, and the stellar yields $M_{y}(\mathrm{He})$ and $M_{y}^{\mathrm{P}}(\mathrm{CO})$ - can either be easily derived from published stellar tracks, or are already tabulated in papers that deal with chemical yields.

Finally, the two components of Eq. (3) can be also distinguished:

$$
\begin{aligned}
\Delta M_{\mathrm{H}} \simeq & X_{0}\left(M_{\mathrm{c}}^{\mathrm{AGBt}}-M_{\mathrm{c}}^{\mathrm{TO}}\right)+M_{y}(\mathrm{He}) \\
& +\left(1-Y^{\prime}\right) M_{y}^{\mathrm{P}}(\mathrm{CO}) \\
\Delta M_{\mathrm{He}} \simeq & M_{\mathrm{c}}^{\mathrm{AGBt}}+M_{y}^{\mathrm{P}}(\mathrm{CO}) .
\end{aligned}
$$

The above equations can be slightly modified when we consider stars (with $M_{\mathrm{i}} \gtrsim 3.5 \quad M_{\odot}$ ) experiencing hotbottom burning (in addition to the dredge-up episodes) during the TP-AGB phase. In this case the dredged-up ${ }^{12} \mathrm{C}$ (and possibly ${ }^{16} \mathrm{O}$ ) may be converted into ${ }^{14} \mathrm{~N}$, so that it is advisable to replace $M_{y}^{\mathrm{P}}(\mathrm{CO})$ in Eqs. (12)-(14) with the total primary yield of the CNO elements, $M_{y}^{\mathrm{P}}(\mathrm{CNO})$.

With the aid of Figs. 1 and 2, we can easily specify the contributions to the total fuel coming from the different post-main sequence shell(s)-burning phases:

$F_{\mathrm{T}}=F_{\mathrm{RGB}}+F_{\mathrm{CHeB}+\mathrm{EAGB}}+F_{\mathrm{TP}-\mathrm{AGB}}$

where the rigt-hand side terms are those derived in the following.

\subsubsection{The RGB fuel}

$$
\begin{aligned}
F_{\mathrm{RGB}} \simeq & \left(X_{1}+0.1\right)\left(M_{\mathrm{c}}^{\mathrm{RGBt}}-M_{\mathrm{c}}^{\mathrm{TO}}\right) \\
& +\left(Y_{1}-Y_{0}\right)\left(M_{\mathrm{i}}-M_{\mathrm{c}}^{\mathrm{RGBt}}\right) .
\end{aligned}
$$

Here, the term related to the 1st dredge-up is given by the amount of newly synthesized helium mixed up into the envelope mass $M_{\mathrm{i}}-M_{\mathrm{c}}^{\mathrm{RGBt}}$, where $M_{\mathrm{i}}$ is the initial stellar mass. We can notice that in this case the term $F_{y}^{\mathrm{CO}}=0$, as the first dredge-up involves material that has experienced only $\mathrm{H}$-burning reactions.

This equation requires the evaluation of $M_{\mathrm{c}}^{\mathrm{RGBt}}$, the core mass at He-ignition. This quantity is normally tabulated only for low-mass stars, because it critically determines the luminosity of $\mathrm{HB}$ and red clump stars. Moreover, in low-mass stars we always have $M_{\mathrm{c}}^{\mathrm{RGBt}}-$ $M_{\mathrm{c}}^{\mathrm{TO}}>0$, since the mass of the electron-degenerate core must grow up to $0.45-0.50 M_{\odot}$ before He can ignite.

For intermediate-mass stars, the RGB phase is practically missing. Anyhow, for the sake of a uniform notation, we can still refer to $F_{\mathrm{RGB}}$ as the fuel (very little) consumed from the TAMS up to central He-ignition. Moreover, we notice that, in general, the quantity $M_{\mathrm{c}}^{\mathrm{RGBt}}$ is not easily singled out in stellar tracks. In fact, in intermediate-mass models the H-burning shell is rather thick (in mass), so that a sharp $\mathrm{H}-\mathrm{He}$ discontinuity cannot be recognised. To overcome this difficulty we may adopt a simple approach, that is to derive $M_{\mathrm{c}}^{\mathrm{RGBt}}$ from:

$M_{\mathrm{c}}^{\mathrm{RGBt}} \simeq M_{\mathrm{c}}^{\mathrm{TO}}-M_{\mathrm{dred}}$, 
where

$M_{\mathrm{dred}} \simeq \frac{Y_{1}-Y_{0}}{1-Y_{1}}\left(M_{\mathrm{i}}-M_{\mathrm{c}}^{\mathrm{TO}}\right)$.

In other words we assume that during the "RGB" phase the core is only affected by the 1st dredge-up, which reduces its mass (by the amount $M_{\mathrm{dred}}=M_{\mathrm{c}}^{\mathrm{TO}}-M_{\mathrm{c}}^{\mathrm{RGBt}}$ ), and increases the envelope helium content from $Y_{0}$ to $Y_{1}$ (normally a tabulated quantity). Actually the 1st dredgeup usually proceeds inward across a chemical profile, instead of a $\mathrm{H}-\mathrm{He}$ discontinuity implicitly assumed in the latter formula. Therefore, the above equations give just a crude approximation of $M_{\mathrm{c}}^{\mathrm{RGBt}}$ for intermediate-mass stars.

\subsubsection{The core He-burning + early-AGB fuel}

$$
\begin{aligned}
F_{\mathrm{CHeB}+\mathrm{EAGB}} \simeq & \left(X_{1}+0.1\right) M_{\mathrm{c}}^{1 \mathrm{TP}}-X_{1} M_{\mathrm{c}}^{\mathrm{RGBt}} \\
& +\left(Y_{2}-Y_{1}\right)\left(M_{\mathrm{i}}-M_{\mathrm{c}}^{1 \mathrm{TP}}\right) .
\end{aligned}
$$

This expression can be easily understood when compared to Eq. (16). The term related to the 2 nd dredge-up is zero whenever this does not occur (i.e. for $M \lesssim 3-4 M_{\odot}$ ).

$M_{\mathrm{c}}^{1 \mathrm{TP}}$ is normally a tabulated quantity, as in e.g. Marigo et al. (1998).

Since core-He burning tends to convert the whole Hecore into a CO-core, and since during most of the E-AGB phase the H-burning shell is off, one can also give a rough estimate to the fuel that comes from the E-AGB phase only:

$$
\begin{aligned}
F_{\mathrm{EAGB}} \sim & 0.1\left(M_{\mathrm{c}}^{1 \mathrm{TP}}-M_{\mathrm{c}}^{\mathrm{RGBt}}\right) \\
& +\left(Y_{2}-Y_{1}\right)\left(M_{\mathrm{i}}-M_{\mathrm{c}}^{1 \mathrm{TP}}\right) .
\end{aligned}
$$

Finally, we remind the reader to consider the cautionary remarks, expressed in Sect. 3.2.2, on the definition of $M_{\mathrm{c}}^{\mathrm{RGBt}}$ in the case of intermediate-mass stars.

\subsubsection{The TP-AGB fuel}

$$
\begin{aligned}
F_{\mathrm{TP}-\mathrm{AGB}} \simeq & \left(X_{1,2}+0.1\right)\left(M_{\mathrm{c}}^{\mathrm{AGBt}}-M_{\mathrm{c}}^{1 \mathrm{TP}}\right) \\
& +M_{y}^{\mathrm{TP}-\mathrm{AGB}}(\mathrm{He}) \\
& +\left(1.1-Y^{\prime}\right) M_{y}^{\mathrm{P}}(\mathrm{CO}) .
\end{aligned}
$$

It should be noticed that the quantity related to the stellar yield of helium, $M_{y}^{\mathrm{TP}-\mathrm{AGB}}(\mathrm{He})$, should be scaled with respect to the elemental abundance at the beginning of the TP-AGB phase, and not to the initial one as in the standard definition of stellar yields (and in Eq. (10)). Then, the ${ }^{4} \mathrm{He}$ contribution can be expressed as

$$
M_{y}^{\mathrm{TP}-\mathrm{AGB}}(\mathrm{He})=\sum_{j=1}^{N_{\mathrm{p}}}\left[\left(Y_{j+2}-Y_{1,2}\right) \Delta M_{j}\right],
$$

where $Y_{j+2}$ corresponds to the abundance after the $j$ th thermal pulse (or $(j+2)$ th-dredge-up event), and $\Delta M_{j}$ denotes the mass ejected during the $j$ th pulse-cycle. The summation is performed over the total number, $N_{\mathrm{p}}$, of pulse cycles. A functional form analogous to Eq. (22) should apply to $M_{y}^{\mathrm{P}}(\mathrm{CO})$ as well, i.e.:

$M_{y}^{\mathrm{P}}(\mathrm{CO})=\sum_{j=1}^{N_{\mathrm{p}}}\left[X^{\mathrm{P}}(\mathrm{C}+\mathrm{O})_{j+2} \Delta M_{j}\right]$,

where $X^{\mathrm{P}}(\mathrm{C}+\mathrm{O})_{j+2}$ denotes the primary carbon and oxygen abundance in the envelope after the $j$ th thermal pulse. It should be noticed that, in this case, the surface chemical composition at the onset of the TP-AGB phase does not contain any element of primary origin, i.e. the scaling term (corresponding to $Y_{1,2}$ in Eq. (22)) is set to zero in Eq. (23).

Finally, we recall that in case of hot-bottom burning a better approximation is achieved using $M_{y}^{\mathrm{P}}(\mathrm{CNO})$ instead of $M_{y}^{\mathrm{P}}(\mathrm{CO})$ (see earlier in this section).

\subsubsection{Further corrections}

The analytical relations presented so far consider the main contributions to the fuel, (i.e. nuclear burnings), but neglect other terms that possibly take part to the energy balance of a star, such as neutrino losses, and energy gains due to gravitational contraction. For the sake of completeness, these terms should be added to the right-hand side of Eq. (8). However, neglecting them does not introduce a significant error in the evaluation of the fuel, as these terms only determine small corrections not exceeding a small percentage in most cases.

Anyhow, we can easily get a rough estimate of the gravitational energy released by gravitational contraction during the evolution of low- and intermediate-mass stars with

$\Delta E_{\mathrm{G}}=-\frac{G M_{\mathrm{WD}}^{2}}{R_{\mathrm{WD}}}$

which gives the order of magnitude of the gravitational energy of a white dwarf with a mass $M_{\mathrm{WD}}$ and radius $R_{\text {WD }}$. This latter can be derived, for instance, adopting the mass-radius relation for white dwarfs (as derived from Tuchman et al. 1983 basing on Chandrasekhar 1939):

$R_{\mathrm{WD}}=0.019\left(1-0.58 M_{\mathrm{WD}}\right)$

(expressed here in solar units) and setting $M_{\mathrm{WD}}=$ $M_{\mathrm{c}}^{\mathrm{AGBt}}$, i.e. the mass of the core left at the end of the AGB.

Then, the corresponding contribution to the stellar fuel (in mass units) can be evaluated with

$\Delta F_{\mathrm{G}}=-\frac{1}{2} \frac{\Delta E_{\mathrm{G}}}{A_{\mathrm{H}}}$

where the factor $1 / 2$ accounts for the fact that only half of the gravitational energy goes in radiation (Virial 
Theorem). A simple evaluation reveals that $\Delta F_{\mathrm{G}}$ is of order of just a few hundredths of $M_{\odot}$. The relative contribution of this term to the total fuel will be shown in a few examples discussed in Sect. 4.

As far as neutrino losses are concerned, in the evolutionary phases under consideration they can be essentially of two kinds:

1. Neutrino losses via $\beta$ decays: they carry away a fraction of the energy produced by nuclear reactions. Since the constant $A_{\mathrm{H}}$ (Eq. (2)) includes only the energy that is effectively available to the star - thus excluding neutrinos - no further correction is needed in our equations;

2. Plasma neutrino losses: they cool the stellar cores during the RGB and AGB phases. However, the energy they carry away is always very small if compared to the stellar surface luminosity. In RGB stars, just some thousandths of the stellar energy leaves the star in the form of plasma neutrinos, whereas in the AGB this fraction is of a few hundredths - reaching a maximum of about 0.06 in the most luminous and massive AGB stars. Thus, they would lead to very small corrections - a few percent in extreme cases - to the fuel, that we will neglect in our approach.

\subsection{The case of massive stars}

Massive stars evolve through successive nuclear burning stages in their interior up to the supernova (SN) explosion, possibly leaving either a neutron star or a black hole as remnant. In comparison to the case of lowand intermediate-mass stars, several factors hamper the derivation of simple and accurate formulas for the fuel consumed by massive stars, including the complex succession of nuclear burnings in the latest stages of hydrostatic core evolution, and the complex energetics of the explosion event.

Nonetheless, we may still provide simple formulas in order to give reasonable estimates to the fuel. First, we can suitably separate the total fuel into two components:

$F_{\mathrm{T}}=F_{\text {pre-SN }}+F_{\mathrm{SN}}$

that refer to the pre-SN, and SN contributions, respectively. In analogy to Eq. (8), the first term can be generalised as:

$$
\begin{aligned}
F_{\mathrm{pre}-\mathrm{SN}} \simeq & X_{0}\left(M_{\mathrm{c}}^{\mathrm{He}}-M_{\mathrm{c}}^{\mathrm{CO}}\right) \\
& \times \sum_{i \geq 2}\left(X_{0}+x_{i, 1}\right)\left(M_{\mathrm{c}}^{i}-M_{\mathrm{c}}^{i+1}\right) \\
& -X_{0} M_{\mathrm{c}}^{\mathrm{TO}}+F_{y}^{\mathrm{He}}+F_{y}^{\mathrm{CO}} .
\end{aligned}
$$

The first term and the summation in the second term account for all the contributions to the fuel involved in the formation of the "onion-skin" structure inside the $\mathrm{H}$-exhausted core. Here the increase of the index $i$ corresponds to more and more advanced nuclear burnings (e.g. $i=1$ refers to H-burning, $i=2$ is related to Heburning, etc.) and $M_{\mathrm{c}}^{i}$ denotes the mass-coordinate of the $i$ th-element exhausted core (e.g. $M_{\mathrm{c}}^{\mathrm{He}}$ for $i=1, M_{\mathrm{c}}^{\mathrm{CO}}$ for $i=2$, etc.). In general, the quantity $x_{i, j}$ is related to the amount of energy generated in the conversion of nuclei $i$ into nuclei $j$, normalised to that provided by the conversion of $\mathrm{H}$ into ${ }^{4} \mathrm{He}$. It can be estimated from the difference between the binding energies per nucleon, $B_{\mathrm{A}}$, that characterize the nuclei involved ( $i=$ final, $j=$ initial):

$x_{i, j}=\frac{B_{\mathrm{A}(i)}-B_{\mathrm{A}(j)}}{B^{4} \mathrm{He}}$

with respect to that of ${ }^{4} \mathrm{He}$ (the main product of $\mathrm{H}$-burning). We can notice that, by definition, $B_{\mathrm{H}}=0$, and that $x_{2,1} \simeq\left(B^{{ }^{12} \mathrm{C}}-B^{4} \mathrm{He}\right) / B^{4} \mathrm{He} \sim 0.1$, as already seen in the previous sections. In other words, $x_{i, 1}$ represents the global efficiency - relative to that of H-burning - of all nuclear burnings (from that of He to the $i$ th-one) which have successively taken place in the shell of mass $\left(M_{\mathrm{c}}^{i}-M_{\mathrm{c}}^{i+1}\right)$.

Finally, the term $-X_{0} M_{\mathrm{c}}^{\mathrm{TO}}+F_{y}^{\mathrm{He}}+F_{y}^{\mathrm{CO}}$ has the same meaning as in Eq. (8), but with a difference: here the chemical yields refer only to those produced during the pre-SN evolution, that is, to the so-called wind yields.

The practical applicability of Eq. (28) is determined by the fact that the quantities $M_{\mathrm{c}}^{\mathrm{He}}, M_{\mathrm{c}}^{\mathrm{CO}}$, and the wind yields, are normally presented in published pre-SN models (see e.g. Maeder 1992; Woosley \& Weaver 1995; Portinari et al. 1998). Conversely, the interior "onion-skin" structure is generally not explicitly given. Anyway, we can use simple approximations to derive lower and upper limits to the second right-hand side term of Eq. (28). A lower limit is obtained neglecting any nuclear conversion into elements heavier than CO nuclei $\left(x_{2,1}=0.1\right)$, so that the term simply reduces to $\left(X_{0}+0.1\right) M_{\mathrm{c}}^{\mathrm{CO}}$. An extreme upper limit corresponds to supposing that the He-exhausted core has been wholly converted into the iron-group elements. In this case, we have $x_{i, 1}=x\left({ }^{12} \mathrm{C} \rightarrow{ }^{56} \mathrm{Fe}\right) \simeq 0.16$ and the second right-hand side term becomes $\left(X_{0}+0.26\right) M_{\mathrm{c}}^{\mathrm{CO}}$.

Therefore, these simple estimates suggest that $F_{\text {pre-SN }}$ should be comprised within a rather narrow range, of width $\sim 0.16 M_{\mathrm{c}}^{\mathrm{CO}}$. This narrowness is determined by the relatively low amount of energy that is available from the conversion of $\mathrm{CO}$ into heavier elements.

Computing $F_{\mathrm{SN}}$ can be far more difficult, because of the complicated energetics involved in the SN explosion. Anyway, a simple order-of-magnitude estimate comes from the conversion of the typical energy irradiated by SNe, $10^{51} \mathrm{erg}$, into the equivalent mass of burnt $\mathrm{H}$ :

$F_{\mathrm{SN}} \sim 0.1 M_{\odot}$.

Therefore, we would expect that usually $F_{\mathrm{SN}} \ll F_{\text {pre-SN }}$.

\section{Illustrative applications}

Here we will apply the analytical formalism derived in the previous section to a few representative examples, in the domain of low- and intermediate-mass stars. First, we will check the accuracy of the formulas, applying them to a 


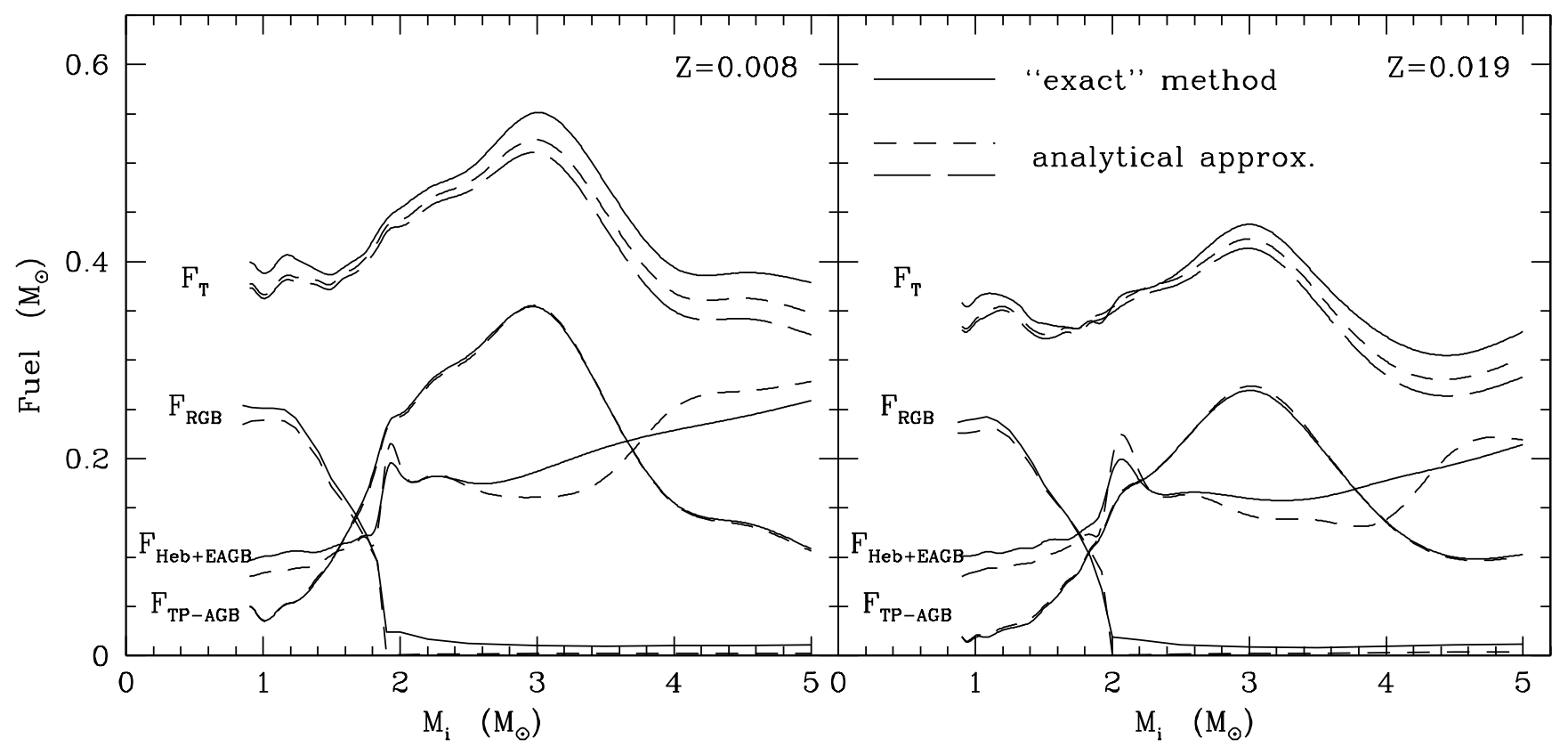

Fig. 3. Accuracy checks. Stellar fuel consumption as a function of the stellar mass, calculated for two sets of stellar models with different initial metallicity (as indicated) and considering various post-MS phases (labelled nearby the corresponding curve). The results predicted by the "exact" method given by Eq. (3) is compared with those obtained by adopting the analytical formalism here proposed (dashed lines). The total fuel is calculated both without (long-dashed line) and with (short-dashed line) the gravitational term expressed by Eq. (26).

particular case in which we know in advance that the stellar inputs are homogeneous and self-consistent (Sect. 4.1). For this specific case, we will analyse the fraction of the fuel that is in the form of chemical yields (Sect. 4.2). This will also allow us to discuss the general features of the fuel as a function of the stellar mass and metallicity, and to quantify the error we may introduce if we neglect the effect of dredge-up episodes (and, in general, of chemical yields) in estimates of the fuel consumption. Finally, we will illustrate a case in which heterogeneous stellar data are used to model the emitted light and chemical yields (Sect. 4.3). This will allow us to remark on the consistency requirements for the input stellar data in chemicalspectrophotometric models of stellar populations.

\subsection{Accuracy checks}

The accuracy of the analytical formalism presented in Sect. 3 should be tested by comparing its predictions to the corresponding results obtained with the "exact" method already mentioned in Sect. 2, which is essentially based on the calculation of the integral in Eq. (5). Of course, this can be done only if we have a homogeneous set of stellar models, providing tables with both (i) the evolutionary tracks (or isochrones), and (ii) the chemical yields and core masses.

Here we adopt a set of low- and intermediate-mass evolutionary models with $0.9 M_{\odot} \lesssim M \leq 5 M_{\odot}, Z_{0}=0.008$, $Y_{0}=0.25$ computed by Girardi et al. (2000) and Marigo et al. (1999), which follow the evolution from the ZAMS up to the end of the AGB, and predict the corresponding stellar yields (Marigo 2001). These models are more extensively described in Appendix A.

The outcome of the test is shown in Fig. 3, for two different initial metallicities $\left(Z_{0}=0.008\right.$ and $\left.Z_{0}=0.019\right)$. It turns out that the analytical prescriptions reproduce remarkably well the fuel calculated with the "exact" method, the relative difference ranging typically within $5 \%$, mostly concerning the RGB, TP-AGB, and the total post-MS contributions. As expected, the largest differences result in the analytical derivation of $F_{\mathrm{RGB}}$ and $F_{\mathrm{CHeB}+\mathrm{EAGB}}$ of intermediate-mass stars, due to the difficulty of defining the core mass-coordinate $M_{\mathrm{c}}^{\mathrm{RGBt}}$ (see remarks in Sect. 3.2.2).

Figure 3 displays also the effect of the correction term, $F_{\mathrm{G}}$, calculated with Eq. (26), which is added to the total fuel derived from Eq. (8). As expected, the convergency towards the "exact" result is improved by a small amount (few percentiles at maximum) with the inclusion of the gravitational term. Finally, we notice that even if some of the partial terms (i.e. $F_{\mathrm{RGB}}, F_{\mathrm{HeB}+\mathrm{EAGB}}$ ) may be not well reproduced, the analytical prescription for the total post-MS fuel $F_{\mathrm{T}}$ is quite accurate. This reflects the small number of stellar parameters involved in Eq. (8).

\subsection{The effect of surface chemical changes}

Figure 4 displays the predicted stellar fuel consumed over the post-main sequence evolution, as a function of the initial stellar mass, again for the Girardi et al. (2000) and Marigo et al. (1999) tracks. For illustrative purposes, we first compute the fuel according to Eq. (8) but 


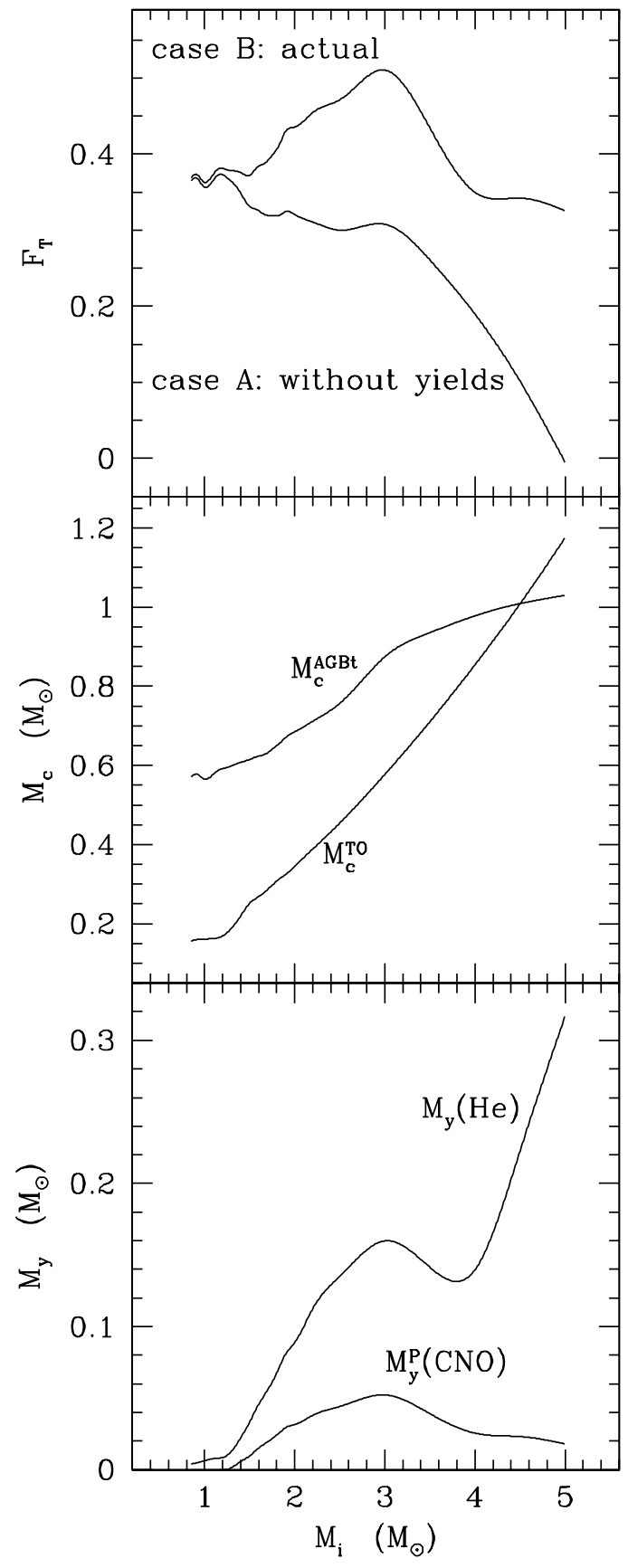

Fig. 4. Upper panel: total post-main sequence fuel consumption by low- and intermediate-mass models with initial composition $\left(Y_{0}=0.25, Z_{0}=0.008\right)$, calculated with and without the terms $\left(F_{y}^{\mathrm{He}}\right.$ and $\left.F_{y}^{\mathrm{CO}}\right)$ related to the chemical yields in Eq. (8). Middle panel: masses of the $\mathrm{H}$-exhausted cores as defined in the text. Bottom panel: chemical yields of ${ }^{4} \mathrm{He}$ and primary CNO. See text for more details.

neglecting the terms due to the dredge-ups (i.e. setting $\left.F_{y}^{\mathrm{He}}=F_{y}^{\mathrm{CO}}=0\right)$. This is referred to as case A. The inclusion of these "chemical" terms corresponds instead to case B, that is the correct derivation of the fuel.

As illustrated in Fig. 4 the differences between the two cases are remarkable for this particular set of stellar models. As expected, the total fuel consumption according to case $\mathrm{A}$ is systematically underestimated with respect to case B. In particular, the following points are worthy of notice.

- The discrepancy - between the results obtained in case $\mathrm{A}$ and the correct ones with prescription $\mathrm{B}$ - increases, on average, with the stellar mass, reflecting the trend of the chemical yields (bottom panel);

- Lower mass stars (with $M \sim 1 M_{\odot}$ ) experience only the first dredge-up during their evolution, so that the "chemical" terms almost do not contribute to their $F_{\mathrm{T}}$;

- At around $M \sim 3 M_{\odot}$ both the actual total fuel $F_{\mathrm{T}}-$ calculated according to case B -, and the yield of helium, $M_{y}(\mathrm{He})$, present a maximum, which corresponds to the longest duration of the TP-AGB phase for this set of stellar models (see Marigo 2001);

- At larger stellar masses, prescription A predicts a drastic drop of the fuel, which does not show up with prescription B. This can be explained comparing the curves of $M_{\mathrm{c}}^{\mathrm{TO}}$ and $M_{\mathrm{c}}^{\mathrm{AGBt}}$ (middle panel of Fig. 4). The difference $\left(M_{\mathrm{c}}^{\mathrm{AGBt}}-M_{\mathrm{c}}^{\mathrm{TO}}\right)$ progressively reduces at increasing stellar mass, eventually becoming negative. In other words, in the most massive models the final cores left at the end of the AGB phase may have even lower masses that the cores built up at the end of the MS. In these cases, the fuel calculated with prescription A would eventually attain negative values, thus losing significance. This example is already a clear indication that the evaluation of the stellar fuel simply basing on core masses is not a correct procedure, as it may even produce meaningless results. Conversely, looking at predictions obtained with prescription B, we can see the total fuel first decreases and then flattens towards the most massive models (keeping always positive), due to the combined effect of both $M_{\mathrm{c}}$ and chemical yields.

The weight of the "chemical terms" can be appreciated also in Fig. 5, which displays the results of the analytical estimation of the fuel consumed during the RGB, TPAGB and total post-MS phases, as a function of the initial stellar mass and metallicity. We can see that the effect of the 1st dredge-up occurring on the RGB is rather small, whereas accounting or not for the surface chemical changes during the AGB leads to quite different results, i.e. the fuel can be substantially underestimated if not doing correctly. Moreover, we notice that the stellar fuel consumption presents a marked metallicity dependence, i.e. it increases, on average, at decreasing metallicity for all the evolutionary phases here considered.

It is also clear that the stellar fuel - calculated with the proposed formalism - is positively correlated to the efficiency of the dredge-up episodes. To give an example, let us consider the TP-AGB evolution of a given stellar model. For the sake of simplicity, we assume that at each thermal pulse a dredge-up occurs with a constant efficiency, $\lambda=\Delta M_{\text {dred }} / \Delta M_{\mathrm{c}}$, defined as the fraction of the core mass increment during an inter-pulse period which is dredgedup into the envelope at the subsequent thermal pulse. This implies that, every time a dredge-up takes place, the core 


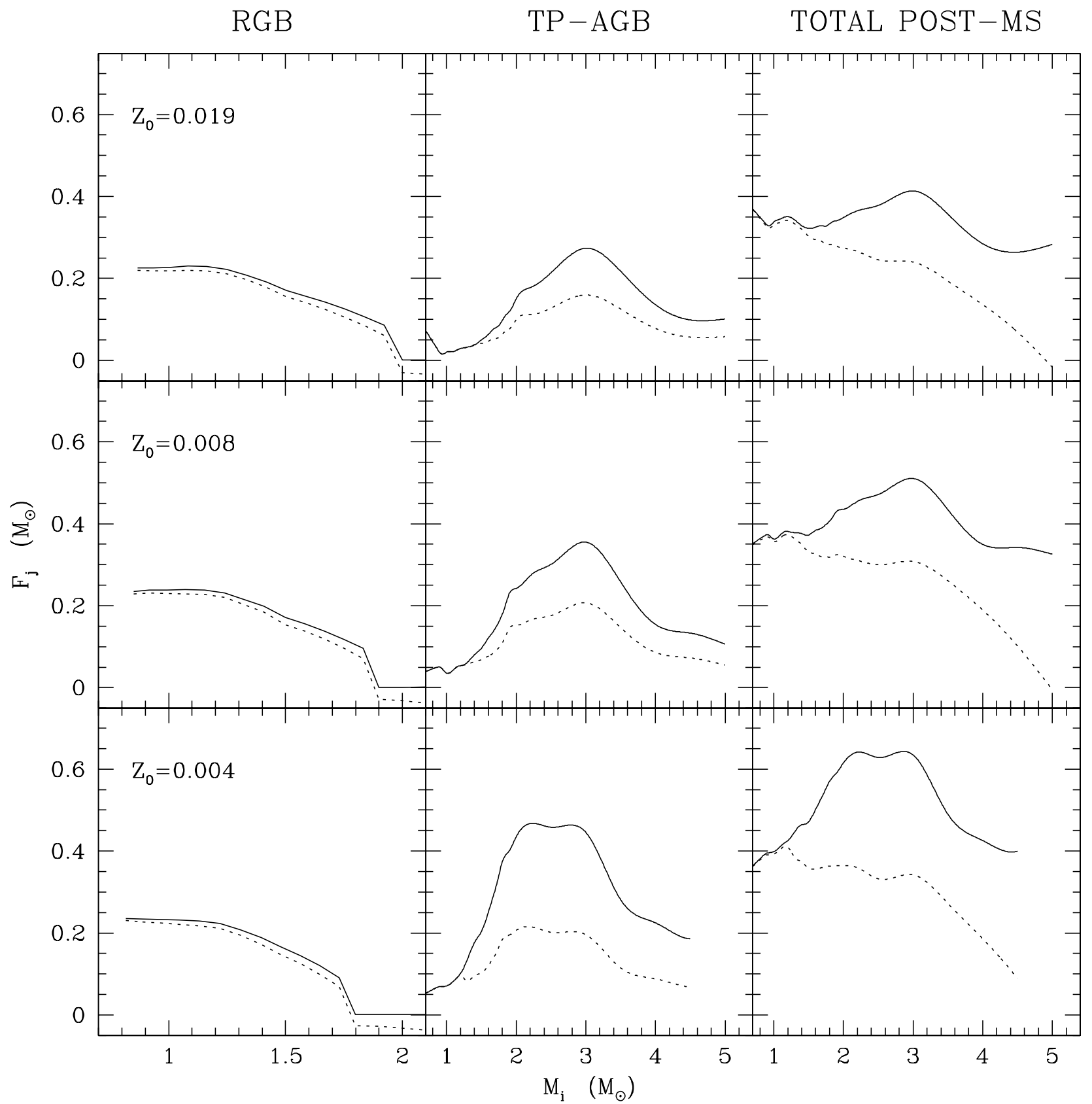

Fig. 5. Stellar fuel consumption (derived with the analytical formalism) of low- and intermediate-mass models with different choices of the initial metallicity, and referring to i) the RGB phase (left panels); TP-AGB phase (middle panels); and total post-MS evolution (right panels). Results of calculations including the effect of dredge-up events and hot-bottom burning (solid lines) are compared to the case in which such effect is neglected (dotted lines). See text for more details.

mass is reduced by the amount $\lambda \Delta M_{\mathrm{c}}$. The TP-AGB fuel consumption can be consequently expressed as:

$F_{\mathrm{TP}-\mathrm{AGB}} \simeq\left(X_{1,2}+0.1\right) \frac{\left(M_{\mathrm{c}}^{\mathrm{AGBt}}-M_{\mathrm{c}}^{1 \mathrm{TP}}\right)}{(1-\lambda)}$

that is equivalent to Eq. (21).

It follows that, if we neglect the effect of third dredgeup, the stellar TP-AGB fuel consumption would be underestimated by a factor $1 /(1-\lambda)$. Setting $\lambda=0.50$, as adopted in the calculations presented in Fig. 4, the underestimation factor is $\sim 2$, i.e. the actual TP-AGB fuel would be twice larger than that one would obtain setting $\lambda=0$. Finally, it is worth noticing that such effect may be even larger if one assumes the extreme dredge-up efficiencies $(\lambda \gtrsim 1)$ of recent TP-AGB calculations (i.e. Herwig $2000)$.

Of course, only case B represents the correct approach to be followed in order to estimate the stellar fuel. However, discussing case A is still useful. In fact, case A represents a sort of lower limit to the fuel, that depends only on the core masses at the MS and AGB termination stages. Since $M_{\mathrm{c}}^{\mathrm{TO}}$ is a monotonic function of the initial 
mass $M_{\mathrm{i}}$, and $M_{\mathrm{c}}^{\mathrm{AGBt}}=M_{\mathrm{WD}}$, it follows that this lower limit is intimately linked to the $M_{\mathrm{WD}}\left(M_{\mathrm{i}}\right)$ relation (see also Girardi \& Bertelli 1998). This latter is known as the initial-final mass relation (IFMR), and is well constrained by observations of nearby white dwarfs (both in the fields and in open clusters). Thus, the observed IFMR poses a direct constraint - i.e. a lower limit - to the behaviour of the stellar fuel as a function of the mass.

In the context of galaxy models, case A can be seen as the fuel (intended as the luminosity integral of Eq. (5)) that we should be dealing with in our spectro-photometric model - instead of the one given by case B - under the assumption that low- and intermediate-mass stars do not contribute to the chemical evolution of the system under consideration. Needless to say, this situation is not realistic at all.

\subsection{The need for a consistency choice in galaxy models}

The analysis and relative discussion carried out in the previous sections should have already convinced the reader on the intimate connection between light emission and chemical yields from stars. Up to now, we have illustrated this fact using a homogeneous set of stellar models (Girardi et al. 2000; Marigo et al. 1999; Marigo 2001). To make concepts even clearer, let us suppose to opt for an inconsistent choice in modelling a galaxy, that is combining the TP-AGB tracks of Marigo (2001, M2K; see Appendix A) to model the spectrophotometric evolution, with the stellar yields of Renzini \& Voli (1981, RV81) to model the chemical evolution ${ }^{4}$.

In this case, we can get two different estimates of the fuel: the first one is derived from our analytical formulas applied to RV81 yields and core masses and corresponds to fuel adopted in the chemical evolution model, and the second one comes from the exact method applied to M2K evolutionary tracks and corresponds to fuel adopted in the spectrophotometric model. Our primary aim is to see how they compare. If the two different inputs were consistent, the two estimates of the fuel should agree to within a few percent, just as in the case of Fig. 3. Otherwise, this exercise would allow us to quantify the degree of mismatch (inconsistency) between these two sources of stellar data.

We use Eq. (21) to derive the fuel consumption of TPAGB models with initial metallicity $Z_{0}=0.02$ presented by RV81. The results are shown in Fig. 6 (left panel). We adopt the final core masses $\left(M_{\mathrm{c}}^{\mathrm{AGBt}}\right)$ and chemical yields presented in Tables $3 \mathrm{f}$ and 3e of RV81, this latter table corresponding to calculations of TP-AGB models (with $\left.M \geq 3.3 M_{\odot}\right)$ with hot-bottom burning carried out with the mixing-length parameter $\alpha=2.0$. For RV81 models,

\footnotetext{
4 Together with those of van den Hoek \& Groenewegen (1997), M2K and RV81 yields are commonly used in chemical evolution models of galaxies. A detailed comparison among the different set of stellar yields is provided in Marigo (2001), to whom we refer for all details.
}

the amounts of mass lost during the RGB phase of lowmass stars (to be properly subtracted from the total yields to get the TP-AGB yields), the envelope abundances of ${ }^{4} \mathrm{He}$ (necessary to calculate the quantities $Y^{\prime}$ ), and the corresponding core masses at the onset of the TP-AGB phase $\left(M_{\mathrm{c}}^{1 \mathrm{TP}}\right)$ are derived following the recipes described in their Sect. 2.

For M2K models with $Z_{0}=0.019$ and $\alpha=1.68$, the TP-AGB fuel has been already presented in our previous Sect. 4.1 (see Fig. 3). The left panel of Fig. 6 shows, instead, M2K fuels corresponding to the same value of $\alpha=2.0$ as adopted in the RV81 models presented in the same figure.

For both sets of stellar models, we also compute the TP-AGB integrated bolometric luminosity as a function of SSP age, according to:

$L_{\mathrm{bol}}^{\mathrm{TP}-\mathrm{AGB}}=A_{\mathrm{H}} b(t) F_{\mathrm{TP}-\mathrm{AGB}}\left(M_{\mathrm{TO}}\right)$

(where all quantities are defined in Sect. 2). They are displayed in the right panel of Fig. 6 .

It is clear, from the comparison between RV81 and M2K results, that the TP-AGB fuel of RV81 models is much larger than the one of M2K models, for masses $M_{\mathrm{i}} \gtrsim$ $3 M_{\odot}$, corresponding to stellar ages shorter than about 300 Myr. More modest but still non-negligible differences are also present at lower masses (older ages).

In addition to the TP-AGB fuel as a function of the initial stellar mass, Fig. 6 (left panel) shows also the contribution of the "chemical" terms to $F_{\mathrm{TP}-\mathrm{AGB}}$ (i.e. the second and third terms in Eq. (21)) for both sets of models. It turns out that i) in both cases the yield contribution to the fuel is generally relevant (except for the lowest mass models that do not experience both the third dredgeup and hot-bottom burning), ii) the differences between RV81 and M2K are still considerable. We can notice that for $M_{\mathrm{i}} \gtrsim 4 M_{\odot}$ the yield contribution to the TP-AGB fuel in RV81 is even larger than the total TP-AGB fuel in M2K models.

As expected, the trend of $F_{\mathrm{TP}-\mathrm{AGB}}$ as a function of $M_{\mathrm{i}}$ (left panel of Fig. 6) reflects in the behaviour of $L_{\mathrm{bol}}^{\mathrm{TP}-\mathrm{AGB}}$ as a function of the corresponding SSP age (for $M_{\mathrm{TO}}=M_{\mathrm{i}}$; right panel of Fig. 6). In fact, here the most relevant differences between RV81 and M2K predictions show up at the youngest ages (hence higher $M_{\mathrm{TO}}$ ), i.e. the luminosity contribution of the TP-AGB phase according to RV81 models is much greater than predicted by M2K models.

On the basis of the above comparative analysis, let us now consider what happens if M2K tracks are used to model the spectro-photometric evolution, whereas RV81 yields are used to model the chemical evolution. The most striking inconsistency in this model would show up at $M_{\mathrm{i}} \sim 4 M_{\odot}$ (or equivalently at $\log t \lesssim 8.4$ ), where the fuel necessary to account for RV81 yields would already be larger than the fuel necessary to account for the luminosity of M2K models. For TP-AGB stars in such a range of masses and ages, our galaxy model we would be either 

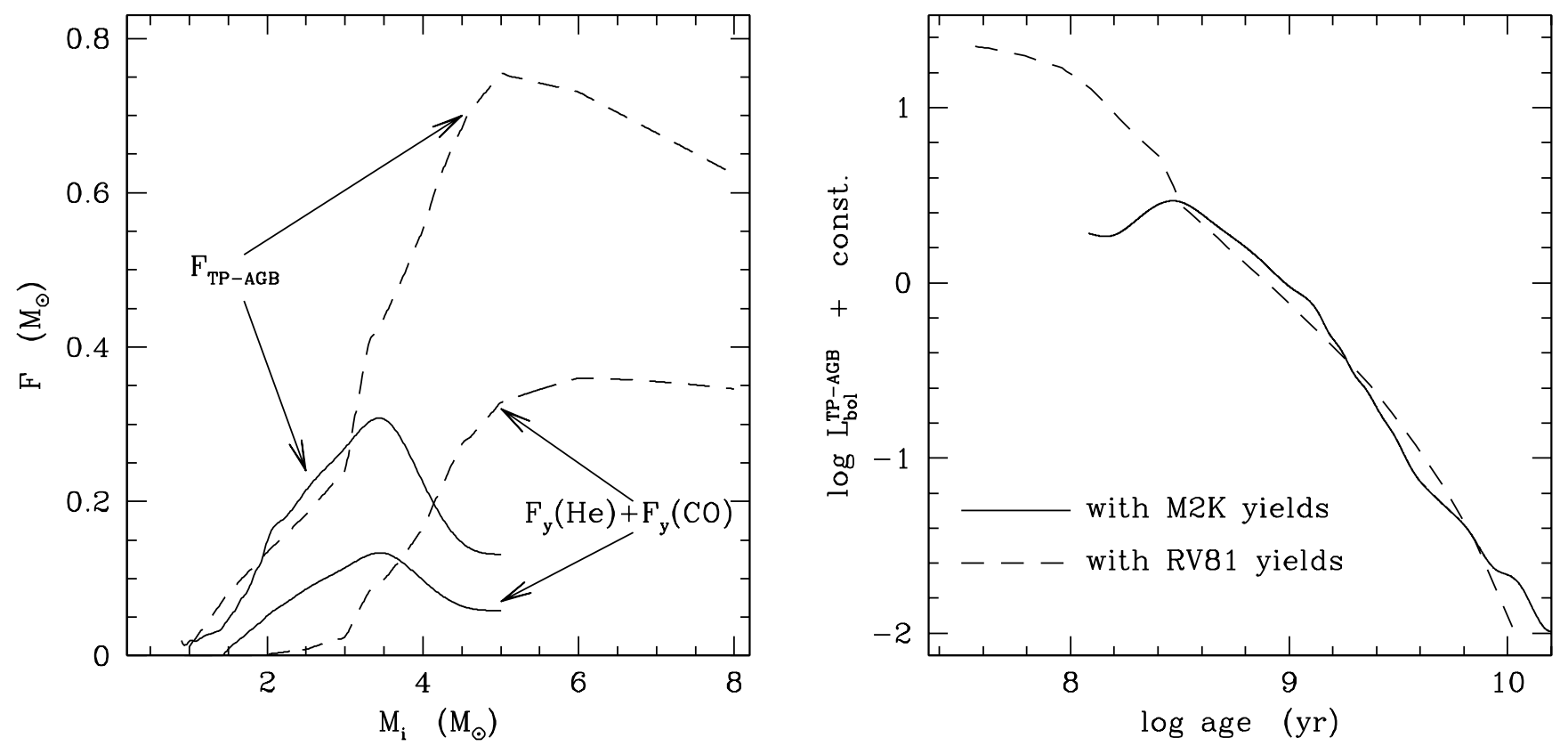

Fig. 6. Left panel: TP-AGB fuel calculated with the analytical formalism here presented and applied to stellar models of Renzini \& Voli (1981, RV81; dashed line), and Marigo (2001, M2K; continuous line). Right panel: contribution (in arbitrary units) of the TP-AGB phase to the integrated bolometric luminosity of SSPs as a function of age. For RV81 models the evolutionary flux $b(t)$ is calculated following Maraston (1998), whereas for M2K models we base on Girardi et al. (2000) evolutionary calculations, in both cases with the assumption of the Salpeter IMF. See text for more details.

strongly overestimating the chemical yields or, equivalently, significantly underestimating the emitted light. The degree of inconsistency is smaller, but still significant, at smaller masses (longer ages).

Since the TP-AGB phase provides a significant fraction of the total energy emitted by stellar populations (meant either as bolometric light, or as chemical yields), one may deduce that such models can hardly be considered as selfconsistent in terms of their energetics.

Moreover, a number of other subtle inconsistencies may affect such a galaxy model. For instance, RV81 and M2K models predict quite different initial-final mass relations (see M2K for a discussion). Whereas a chemical model that uses RV81 yields is expected to produce a relatively large fraction of "massive" white dwarfs (with $\left.M_{\mathrm{WD}} \sim 1 M_{\odot}\right)$, a spectro-photometric model that adopts M2K tracks, under the same assumptions for star formation rate and age-metallicity relation, is expected to predict much fewer of them. Then, in view of modelling, for instance, the formation of galactic halos one would face the embarrassing question: which one of the two theoretical white dwarf mass distributions should be considered for a comparison with the observational data of field WDs and MACHOs? Any is the answer, it will be the wrong one.

\section{Final remarks}

This study has highlighted the intimate relation between the stellar emitted light and chemical yields. It has been clarified that a significant fraction of fuel consumption (emitted light) may be eventually "deposited" in the form of $\mathrm{He}$ and $\mathrm{CO}$ yields. This fraction can be quantified by means of simple analytical relations. We have illustrated this in detail for low- and intermediate-mass stars, also suggesting how the same analysis could be extended to massive stars. Then, the derived formalism offers a useful consistency check that can be applied to galaxy models whenever different sources of stellar data are employed to model the chemical and spectrophotometric evolution.

Such consistency check expresses essentially the basic condition of energy conservation, which can be read as: the emitted light implies a precise amount of stellar nucleosynthesis, part of which corresponds to chemical yields. Other fundamental aspects, such as mass conservation, are already included in the basic equations of chemical evolution models.

It is important to remark that the present analysis is general, and independent of the specific set of stellar tracks/yields in use. As a matter of fact, analysing the quality of any given set of stellar (or galaxy) models, is beyond the scope of this paper.

Anyway, a few remarks can be made. As indicated by our analysis, the primary yields of He and CNO elements are those more directly related to the emitted light of a stellar population. In galactic chemical evolution models, He and CNO yields critically determine the evolution of $Y(t)$ (or, equivalently, the $\Delta Y / \Delta Z$ ratio) and $Z(t)$ relations. Our formalism suggests that, if a given set of stellar models predicts a particular evolution of the integrated light, the same set of models necessarily implies a particular evolution of $Y(t)$ and $Z(t)$. 
Of course, the situation is somewhat complicated by the fact that most of the metals are produced by massive stars, which are characterised by rapid evolution, so that soon they do not contribute to the integrated light anymore. Hence, light and chemical evolution may be dominated by different stars, possibly justifying the approximation that photometric and chemical evolution could be treated separately. Actually, this assumption may be safely applied to some cases (e.g. in the study of the present Galaxy), but becomes questionable (and risky) once galaxy models are constructed to describe the evolution of galaxies over most of their history, since their formation up to present times. An example of this "unifying" approach is given by the studies of high-redshift galaxies based on models "calibrated" on local galaxy samples. Clearly, our analysis indicates that the energetics necessary to explain the light of the distant sample has strict implications for the chemistry now observed in the local sample, and vice-versa.

Finally, we would like to conclude outlining the main points of the present work:

- The stellar fuel (emitted light) consumed by a star during a specified evolutionary phase may be schematically decomposed as the sum of two terms: a contribution locked in the star and related to the mass of the core regions and envelope composition, and a contribution emitted by the star during that phase in the form of chemical yields. These terms are explicited in this paper by means of suitable and simple analytical formulas, covering various phases of stellar evolution;

- When coupling the spectro-photometric and chemical evolution of a system, we should care that the basic contributions of the stellar component - i.e. light and newly synthesised elements - are included in the models consistently one with each other, preferably derived from the same set of stellar models. If this is not the case, i.e. the input stellar data come from heterogeneous sources, we could at least measure the degree of internal mismatch of the model. The analytical formalism developed in this work can serve the purpose.

Acknowledgements. L. G. thanks Martin Groenewegen for very useful conversations about the third dredge-up. We warmly thank Elena Fantino for her invaluable help in the preparation of the figures. This work was partly funded by the Italian Ministry of University, Scientific Research and Technology (MURST).

\section{Appendix A: Isochrones with improved TP-AGB models}

Girardi et al. (2000) presented a large set of evolutionary tracks for low- and intermediate-mass stars, covering the mass range from 0.15 to $7 M_{\odot}$, for six different initial chemical compositions. These models account for a moderate amount of convective overshooting from stellar cores, which makes the upper mass limit for the development of the AGB phase to be about $5 M_{\odot}$, instead of $8 M_{\odot}$ as predicted by classical stellar models.

Since the evolutionary calculations, carried out by means of the complete stellar code, stopped after the first few thermal pulses, Girardi et al. (2000) followed the subsequent TP-AGB phase with the aid of a simple synthetic algorithm (cf. Girardi \& Bertelli 1998). In this way, sets of isochrones including the complete TP-AGB phase were presented.

That approach to the TP-AGB evolution, however, is far too simple if compared to the detailed semi-analytical TP-AGB models presented by Marigo et al. (1999, and references therein). These latter models include the most crucial aspects of TP-AGB evolution, such as the third dredge-up, hot-bottom burning, deviations from the core mass-luminosity relation, and mass-loss rates related to the pulsational period. Moreover, the basic model parameters have been calibrated such as to reproduce the carbon star luminosity functions in the LMC and SMC. Chemical yields from these models are presented by Marigo (2001).

Marigo et al. (1999) tracks start at the first thermal pulse computed by Girardi et al. (2000), so that both sets of models are perfectly complementary. They follow the entire TP-AGB evolution up to the complete ejection of the stellar envelope. These synthetic TP-AGB tracks are available, so far, only for chemical compositions $\left[Z_{0}=0.004, Y_{0}=0.240\right],\left[Z_{0}=0.008, Y_{0}=0.250\right]$ and $\left[Z_{0}=0.019, Y_{0}=0.273\right]$. For these metallicities, the synthetic TP-AGB models have been suitably combined to the tracks calculated by Girardi et al. (2000), so that theoretical isochrones have been constructed using the same method as in Girardi et al. (2000).

Thanks to their continuity and homogeneity, the present isochrones are well suited for the population synthesis of galaxies. Moreover, they perfectly meet the consistency requirements discussed in this paper: the emitted light computed with the present isochrones (with the aid of Eq. (1)), would be completely consistent with the chemical evolution derived from the tables of chemical yields and remnant masses in Marigo (2001).

Moreover, it is worth emphasizing that Marigo et al. (1999) models reproduce a series of observational properties of AGB stars in nearby galaxies, and of nearby white dwarfs (see Marigo 2001 for details). Therefore, one may expect that they are adequate to model the integrated light emitted by TP-AGB stars.

\section{A.1. Integrated ligth contribution from AGB stars}

In order to check this latter point, we compare the present models to the data from Frogel et al. (1990), who measured the contribution of luminous AGB stars to the integrated light of Magellanic Cloud star clusters of various ages. To make the comparison, we proceed as follow: first, from their Table 1 we compute the bolometric light coming from stars with $M_{\mathrm{bol}}<-3.8$ in each cluster (assuming distance moduli of 18.5 and 18.9 mag for the LMC and SMC, 


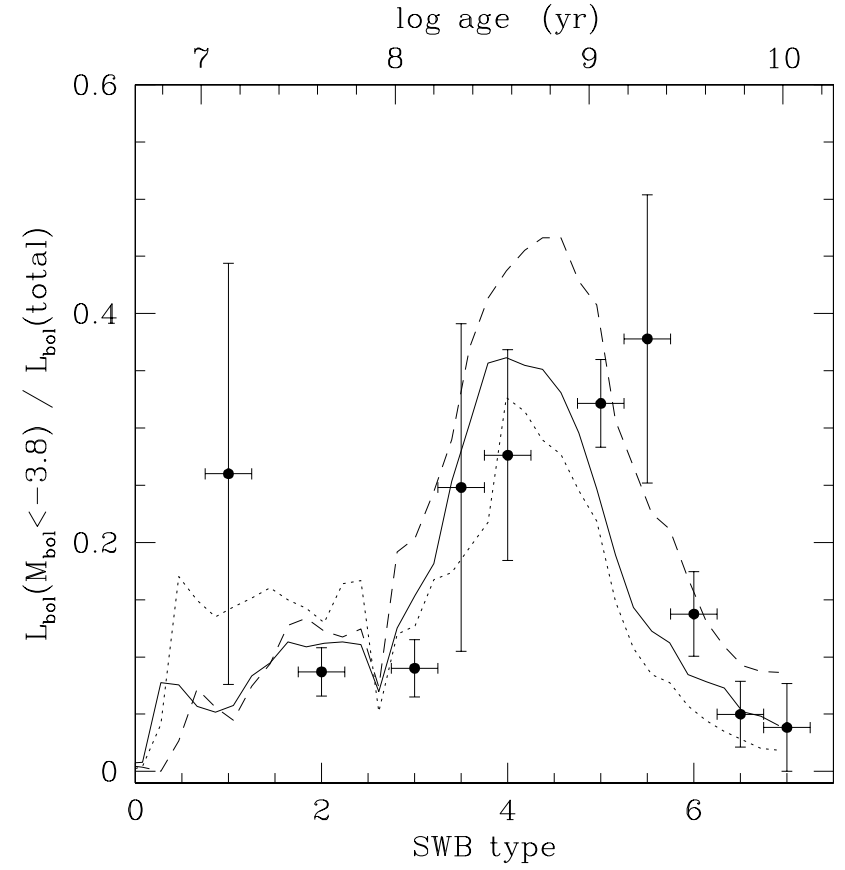

Fig. A.1. The fractional contribution of luminous AGB stars tho the integrated light of LMC and SMC star clusters, as inferred from Frogel et al. (1990) data (full dots with error bars), as a function of the equivalent SWB types. This latter represents a rough age ranking for LMC and SMC clusters, that corresponds to the logarithmic age scale presented in the upper axe. The same quantity is plotted, as a function of age, as derived from our isochrones of metallicity $Z_{0}=0.004$ (dashed line), $Z_{0}=0.008$ (continuous line) and $Z_{0}=0.019$ (dotted line).

respectively). This $M_{\text {bol }}$ limit guarantees that we are dealing only with AGB stars brighter than the RGBtip. Second, we divide the clusters into bins of equivalent SWB (Searle et al. 1980) types (cf. Table 4 in Frogel et al.). Third, we compute the ratio, $L_{\mathrm{bol}}\left(M_{\mathrm{bol}}<\right.$ $-3.8) / L_{\text {bol }}$ (total), between the luminosity coming from stars with $M_{\text {bol }}<-3.8$ and that emitted from the whole cluster (cf. their Table 4). Finally, we estimate the corresponding error bars from the total number of $M_{\text {bol }}<-3.8$ stars in each SWB bin.

The results are presented in Fig. A.1, which also displays the approximate relation between SWB type and the logarithm of cluster age, i.e. $\log (t / \mathrm{yr}) \simeq 6.66+0.48 \mathrm{SWB}$. The observed data in this figure are indeed very similar to the original ones in Fig. 15 of Frogel et al. (1990). Superimposed on the data, we plot our theoretical expectation for the same quantity, $L_{\mathrm{bol}}\left(M_{\mathrm{bol}}<\right.$ $-3.8) / L_{\mathrm{bol}}$ (total), as derived from the present isochrones.

From the figure, it is well evident that the models correctly reproduce the general behaviour of the relative luminosity contribution over the complete age interval in which AGB stars are present (i.e. $8 \lesssim \log (t / \mathrm{yr}) \lesssim 10$ ). In particular, the minimum values at $\log (t / \mathrm{yr}) \sim 8$ and $\sim 10$ are clearly present in the models, as well as the maximum at $\log (t / y r) \sim 8.6$, that would correspond to the
Table A1. Post-MS stellar fuels $\left(M_{\odot}\right)-Z_{0}=0.019$.

\begin{tabular}{ccccc}
\hline$M_{\mathrm{i}}$ & $F_{\text {RGB }}$ & $F_{\text {CHeb+E-AGB }}$ & $F_{\text {TP-AGB }}$ & $F_{\mathrm{T}}$ \\
\hline 0.868 & $2.371 \mathrm{E}-01$ & $1.001 \mathrm{E}-01$ & $2.892 \mathrm{E}-02$ & $3.661 \mathrm{E}-01$ \\
0.934 & $2.387 \mathrm{E}-01$ & $1.014 \mathrm{E}-01$ & $1.400 \mathrm{E}-02$ & $3.542 \mathrm{E}-01$ \\
1.005 & $2.405 \mathrm{E}-01$ & $1.023 \mathrm{E}-01$ & $1.912 \mathrm{E}-02$ & $3.620 \mathrm{E}-01$ \\
1.082 & $2.428 \mathrm{E}-01$ & $1.059 \mathrm{E}-01$ & $1.887 \mathrm{E}-02$ & $3.675 \mathrm{E}-01$ \\
1.163 & $2.385 \mathrm{E}-01$ & $1.042 \mathrm{E}-01$ & $2.402 \mathrm{E}-02$ & $3.667 \mathrm{E}-01$ \\
1.248 & $2.287 \mathrm{E}-01$ & $1.061 \mathrm{E}-01$ & $2.778 \mathrm{E}-02$ & $3.625 \mathrm{E}-01$ \\
1.334 & $2.141 \mathrm{E}-01$ & $1.091 \mathrm{E}-01$ & $3.049 \mathrm{E}-02$ & $3.537 \mathrm{E}-01$ \\
1.420 & $1.951 \mathrm{E}-01$ & $1.095 \mathrm{E}-01$ & $3.577 \mathrm{E}-02$ & $3.405 \mathrm{E}-01$ \\
1.504 & $1.731 \mathrm{E}-01$ & $1.158 \mathrm{E}-01$ & $4.829 \mathrm{E}-02$ & $3.372 \mathrm{E}-01$ \\
1.588 & $1.573 \mathrm{E}-01$ & $1.182 \mathrm{E}-01$ & $5.916 \mathrm{E}-02$ & $3.347 \mathrm{E}-01$ \\
1.672 & $1.415 \mathrm{E}-01$ & $1.179 \mathrm{E}-01$ & $7.404 \mathrm{E}-02$ & $3.334 \mathrm{E}-01$ \\
1.756 & $1.230 \mathrm{E}-01$ & $1.245 \mathrm{E}-01$ & $8.503 \mathrm{E}-02$ & $3.326 \mathrm{E}-01$ \\
1.839 & $1.006 \mathrm{E}-01$ & $1.327 \mathrm{E}-01$ & $1.065 \mathrm{E}-01$ & $3.398 \mathrm{E}-01$ \\
1.923 & $6.746 \mathrm{E}-02$ & $1.483 \mathrm{E}-01$ & $1.222 \mathrm{E}-01$ & $3.380 \mathrm{E}-01$ \\
2.000 & $1.910 \mathrm{E}-02$ & $1.870 \mathrm{E}-01$ & $1.485 \mathrm{E}-01$ & $3.546 \mathrm{E}-01$ \\
2.200 & $1.640 \mathrm{E}-02$ & $1.781 \mathrm{E}-01$ & $1.766 \mathrm{E}-01$ & $3.711 \mathrm{E}-01$ \\
2.500 & $1.130 \mathrm{E}-02$ & $1.652 \mathrm{E}-01$ & $2.145 \mathrm{E}-01$ & $3.909 \mathrm{E}-01$ \\
3.000 & $8.800 \mathrm{E}-03$ & $1.597 \mathrm{E}-01$ & $2.693 \mathrm{E}-01$ & $4.378 \mathrm{E}-01$ \\
3.500 & $8.600 \mathrm{E}-03$ & $1.604 \mathrm{E}-01$ & $2.149 \mathrm{E}-01$ & $3.839 \mathrm{E}-01$ \\
4.000 & $9.700 \mathrm{E}-03$ & $1.780 \mathrm{E}-01$ & $1.363 \mathrm{E}-01$ & $3.239 \mathrm{E}-01$ \\
4.500 & $1.090 \mathrm{E}-02$ & $1.944 \mathrm{E}-01$ & $1.001 \mathrm{E}-01$ & $3.052 \mathrm{E}-01$ \\
5.000 & $1.170 \mathrm{E}-02$ & $2.144 \mathrm{E}-01$ & $1.030 \mathrm{E}-01$ & $3.289 \mathrm{E}-01$ \\
\hline & & & &
\end{tabular}

longest TP-AGB lifetimes (see Marigo 2001). It is also worth noticing that according to the models at decreasing metallicity the luminosity contribution from AGB stars increases.

For ages younger than $10^{8} \mathrm{yr}$, we have computed the same quantities using Bertelli et al. (1994) isochrones. The integration of $L_{\mathrm{bol}}\left(M_{\mathrm{bol}}<-3.8\right)$ was limited to stars with $\log T_{\text {eff }}<3.8$, in order to exclude the blue main sequence stars from this term. As shown in Fig. A.1, the models predict values $L_{\mathrm{bol}}\left(M_{\mathrm{bol}}<-3.8\right) / L_{\mathrm{bol}}($ total $)$ of around 0.1 at these young ages. They are in reasonable agreement with observations, despite of the fact that the empirical data present a slight hint for an increase in $L_{\mathrm{bol}}\left(M_{\mathrm{bol}}<\right.$ $-3.8)$ at even younger ages $\left(\sim 10^{7} \mathrm{yr}\right)$ (we notice, however, this feature is based on too few stars to be completely reliable).

With the cautionary remarks that (i) the SWB classification represents just a crude age ranking for clusters, whose consistency with our models has not been checked for, and (ii) that there is plenty of room for improving the empirical data for AGB stars in clusters, we conclude that our models reproduce the observed contribution of AGB stars to the integrated light of SSPs in a satisfactory way.

\section{A.2. Useful data}

Complete tables with the improved isochrones described in the previous section are available upon request to the authors, and at the WWW site http://pleiadi.pd.astro.it.

For the present models, Tables A1-A3 contain the stellar fuels as a function of the initial mass, for various 
Table A2. Post-MS stellar fuels $\left(M_{\odot}\right)-Z_{0}=0.008$.

\begin{tabular}{ccccc}
\hline$M_{\mathrm{i}}$ & $F_{\mathrm{RGB}}$ & $F_{\mathrm{CHeb}+\mathrm{E}-\mathrm{AGB}}$ & $F_{\mathrm{TP}-\mathrm{AGB}}$ & $F_{\mathrm{T}}$ \\
\hline 0.850 & $2.542 \mathrm{E}-01$ & $9.550 \mathrm{E}-02$ & $4.851 \mathrm{E}-02$ & $3.982 \mathrm{E}-01$ \\
0.918 & $2.522 \mathrm{E}-01$ & $9.746 \mathrm{E}-02$ & $4.888 \mathrm{E}-02$ & $3.986 \mathrm{E}-01$ \\
0.992 & $2.516 \mathrm{E}-01$ & $1.006 \mathrm{E}-01$ & $3.666 \mathrm{E}-02$ & $3.889 \mathrm{E}-01$ \\
1.071 & $2.514 \mathrm{E}-01$ & $1.017 \mathrm{E}-01$ & $4.052 \mathrm{E}-02$ & $3.936 \mathrm{E}-01$ \\
1.154 & $2.499 \mathrm{E}-01$ & $1.048 \mathrm{E}-01$ & $5.144 \mathrm{E}-02$ & $4.061 \mathrm{E}-01$ \\
1.239 & $2.414 \mathrm{E}-01$ & $1.066 \mathrm{E}-01$ & $5.500 \mathrm{E}-02$ & $4.030 \mathrm{E}-01$ \\
1.407 & $2.061 \mathrm{E}-01$ & $1.055 \mathrm{E}-01$ & $7.898 \mathrm{E}-02$ & $3.906 \mathrm{E}-01$ \\
1.499 & $1.800 \mathrm{E}-01$ & $1.101 \mathrm{E}-01$ & $9.679 \mathrm{E}-02$ & $3.867 \mathrm{E}-01$ \\
1.583 & $1.626 \mathrm{E}-01$ & $1.138 \mathrm{E}-01$ & $1.172 \mathrm{E}-01$ & $3.936 \mathrm{E}-01$ \\
1.667 & $1.452 \mathrm{E}-01$ & $1.168 \mathrm{E}-01$ & $1.392 \mathrm{E}-01$ & $4.012 \mathrm{E}-01$ \\
1.750 & $1.235 \mathrm{E}-01$ & $1.224 \mathrm{E}-01$ & $1.644 \mathrm{E}-01$ & $4.102 \mathrm{E}-01$ \\
1.832 & $9.532 \mathrm{E}-02$ & $1.347 \mathrm{E}-01$ & $1.985 \mathrm{E}-01$ & $4.285 \mathrm{E}-01$ \\
1.900 & $2.450 \mathrm{E}-02$ & $1.866 \mathrm{E}-01$ & $2.317 \mathrm{E}-01$ & $4.427 \mathrm{E}-01$ \\
2.000 & $2.380 \mathrm{E}-02$ & $1.850 \mathrm{E}-01$ & $2.451 \mathrm{E}-01$ & $4.540 \mathrm{E}-01$ \\
2.200 & $1.720 \mathrm{E}-02$ & $1.808 \mathrm{E}-01$ & $2.758 \mathrm{E}-01$ & $4.738 \mathrm{E}-01$ \\
2.500 & $1.260 \mathrm{E}-02$ & $1.759 \mathrm{E}-01$ & $3.047 \mathrm{E}-01$ & $4.933 \mathrm{E}-01$ \\
3.000 & $1.020 \mathrm{E}-02$ & $1.871 \mathrm{E}-01$ & $3.539 \mathrm{E}-01$ & $5.513 \mathrm{E}-01$ \\
3.500 & $9.700 \mathrm{E}-03$ & $2.116 \mathrm{E}-01$ & $2.581 \mathrm{E}-01$ & $4.794 \mathrm{E}-01$ \\
4.000 & $1.030 \mathrm{E}-02$ & $2.288 \mathrm{E}-01$ & $1.552 \mathrm{E}-01$ & $3.943 \mathrm{E}-01$ \\
4.500 & $1.080 \mathrm{E}-02$ & $2.426 \mathrm{E}-01$ & $1.354 \mathrm{E}-01$ & $3.888 \mathrm{E}-01$ \\
5.000 & $1.150 \mathrm{E}-02$ & $2.589 \mathrm{E}-01$ & $1.083 \mathrm{E}-01$ & $3.787 \mathrm{E}-01$ \\
\hline
\end{tabular}

Table A3. Post-MS stellar fuels $\left(M_{\odot}\right)-Z_{0}=0.004$.

\begin{tabular}{ccccc}
\hline$M_{\mathrm{i}}$ & $F_{\mathrm{RGB}}$ & $F_{\mathrm{CHeb}+\mathrm{E}-\mathrm{AGB}}$ & $F_{\mathrm{TP}-\mathrm{AGB}}$ & $F_{\mathrm{T}}$ \\
\hline 0.817 & $2.536 \mathrm{E}-01$ & $9.664 \mathrm{E}-02$ & $5.876 \mathrm{E}-02$ & $4.089 \mathrm{E}-01$ \\
0.888 & $2.527 \mathrm{E}-01$ & $1.005 \mathrm{E}-01$ & $6.502 \mathrm{E}-02$ & $4.182 \mathrm{E}-01$ \\
0.966 & $2.517 \mathrm{E}-01$ & $1.040 \mathrm{E}-01$ & $6.470 \mathrm{E}-02$ & $4.204 \mathrm{E}-01$ \\
1.046 & $2.499 \mathrm{E}-01$ & $1.067 \mathrm{E}-01$ & $7.085 \mathrm{E}-02$ & $4.274 \mathrm{E}-01$ \\
1.131 & $2.467 \mathrm{E}-01$ & $1.091 \mathrm{E}-01$ & $8.151 \mathrm{E}-02$ & $4.373 \mathrm{E}-01$ \\
1.218 & $2.397 \mathrm{E}-01$ & $1.115 \mathrm{E}-01$ & $9.972 \mathrm{E}-02$ & $4.510 \mathrm{E}-01$ \\
1.307 & $2.223 \mathrm{E}-01$ & $1.122 \mathrm{E}-01$ & $1.392 \mathrm{E}-01$ & $4.736 \mathrm{E}-01$ \\
1.396 & $2.002 \mathrm{E}-01$ & $1.147 \mathrm{E}-01$ & $1.771 \mathrm{E}-01$ & $4.919 \mathrm{E}-01$ \\
1.481 & $1.741 \mathrm{E}-01$ & $1.153 \mathrm{E}-01$ & $1.989 \mathrm{E}-01$ & $4.882 \mathrm{E}-01$ \\
1.564 & $1.508 \mathrm{E}-01$ & $1.198 \mathrm{E}-01$ & $2.360 \mathrm{E}-01$ & $5.066 \mathrm{E}-01$ \\
1.647 & $1.246 \mathrm{E}-01$ & $1.275 \mathrm{E}-01$ & $2.829 \mathrm{E}-01$ & $5.351 \mathrm{E}-01$ \\
1.729 & $8.349 \mathrm{E}-02$ & $1.489 \mathrm{E}-01$ & $3.315 \mathrm{E}-01$ & $5.639 \mathrm{E}-01$ \\
1.800 & $2.790 \mathrm{E}-02$ & $1.868 \mathrm{E}-01$ & $3.796 \mathrm{E}-01$ & $5.943 \mathrm{E}-01$ \\
1.900 & $2.390 \mathrm{E}-02$ & $1.824 \mathrm{E}-01$ & $4.073 \mathrm{E}-01$ & $6.136 \mathrm{E}-01$ \\
2.000 & $1.870 \mathrm{E}-02$ & $1.823 \mathrm{E}-01$ & $4.433 \mathrm{E}-01$ & $6.443 \mathrm{E}-01$ \\
2.200 & $1.330 \mathrm{E}-02$ & $1.851 \mathrm{E}-01$ & $4.784 \mathrm{E}-01$ & $6.768 \mathrm{E}-01$ \\
2.500 & $1.010 \mathrm{E}-02$ & $1.939 \mathrm{E}-01$ & $4.713 \mathrm{E}-01$ & $6.753 \mathrm{E}-01$ \\
3.000 & $8.700 \mathrm{E}-03$ & $2.202 \mathrm{E}-01$ & $4.572 \mathrm{E}-01$ & $6.862 \mathrm{E}-01$ \\
3.500 & $9.100 \mathrm{E}-03$ & $2.398 \mathrm{E}-01$ & $2.872 \mathrm{E}-01$ & $5.361 \mathrm{E}-01$ \\
4.000 & $1.000 \mathrm{E}-02$ & $2.497 \mathrm{E}-01$ & $2.324 \mathrm{E}-01$ & $4.922 \mathrm{E}-01$ \\
4.500 & $1.120 \mathrm{E}-02$ & $2.638 \mathrm{E}-01$ & $1.937 \mathrm{E}-01$ & $4.688 \mathrm{E}-01$ \\
5.000 & $1.260 \mathrm{E}-02$ & $2.797 \mathrm{E}-01$ & $2.389 \mathrm{E}-01$ & $5.312 \mathrm{E}-01$ \\
\hline & & & &
\end{tabular}

post-MS phases. All fuels have been calculated by means of Eq. (5).

We also present fitting relations to $M_{\mathrm{c}}^{\mathrm{TO}}$ as a function of the initial mass $M_{\mathrm{i}}$ and metallicity $Z_{0}$, derived from the stellar models here considered (with overshooting):

$M_{\mathrm{c}}^{\mathrm{TO}} \simeq 0.463-0.654 M_{\mathrm{i}}+0.338 M_{\mathrm{i}}^{2}$

$$
\begin{array}{rlr} 
& -0.024 \log \left(Z_{0} / 0.019\right) & \text { for } M_{\mathrm{i}} \lesssim 1.4 \\
M_{\mathrm{c}}^{\mathrm{TO}} \simeq & 0.006+0.115 M_{\mathrm{i}}+0.023 M_{\mathrm{i}}^{2} & \\
& -0.057 \log \left(Z_{0} / 0.019\right) & \text { for } M_{\mathrm{i}} \gtrsim 1.4,
\end{array}
$$

and from classical models (without overshooting) with initial solar composition $\left(Y_{0}=0.273, Z_{0}=0.019\right)$ :

$M_{\mathrm{c}}^{\mathrm{TO}} \simeq 0.123+0.066 M_{\mathrm{i}}-0.037 M_{\mathrm{i}}^{2}$ for $M_{\mathrm{i}} \lesssim 1.4$

$M_{\mathrm{c}}^{\mathrm{TO}} \simeq 0.015+0.063 M_{\mathrm{i}}+0.019 M_{\mathrm{i}}^{2}$ for $M_{\mathrm{i}} \gtrsim 1.4$. (A.4)

All masses are expressed in solar units. We remind that according to our definition of $M_{\mathrm{c}}^{\mathrm{TO}}$ (see Eq. (7)), the fuel burnt during the MS can be estimated from:

$F_{\mathrm{MS}}=X_{0} M_{\mathrm{c}}^{\mathrm{TO}}$.

\section{References}

Arimoto, N., \& Yoshii, Y. 1986, A\&A, 164, 260

Arimoto, N., \& Yoshii, Y. 1987, A\&A, 173, 23

Bertelli, G., Bressan, A., Chiosi, C., Fagotto, F., \& Nasi, E. 1994, A\&AS, 106, 275

Bressan, A., Chiosi, C., \& Fagotto, F. 1994, ApJS, 94, 63

Chabrier, G. 1999, ApJ, 513, L103

Chandrasekhar, S. 1939, An Introduction to the Study of Stellar Structure (University of Chicago Press, Chicago)

Charlot, S., \& Silk, J. 1995, ApJ, 445, 124

Chiosi, C., Bressan, A., Portinari, L., \& Tantalo, R. 1998, A\&A, 339, 355

Einsel, C., Fritze-von Alvensleben, U., Krueger, H., \& Fricke, K. J. 1995, A\&A, 296, 347

Fall, S. M. 2001, in The Extragalactic Infrared Background and its Cosmological Implications, IAU Symp. 204, ed. M. Harwit, \& M. G. Hauser, in press [astro-ph/0101084]

Ferreras, I., \& Silk, J. 2000, MNRAS, 316, 786

Fields, B. D., Freese, K., \& Graff, D. S. 2000, ApJ, 534, 265

Frogel, J. A., Mould, J., \& Blanco, V. M. 1990, ApJ, 352, 96

Girardi, L., \& Bertelli, G. 1998, MNRAS, 300, 533

Girardi, L., Bressan, A., Bertelli, G., \& Chiosi, C. 2000, A\&AS, 141,371

Greggio, L., \& Renzini, A. 1990, ApJ, 364, 35

Herwig, F. 2000, A\&A, 360, 952

Ibata, R., Irwin, M., Bienaymé, O., Scholz, R., \& Guibert, J. 2000, ApJ, 532, L41

Madau, P., \& Pozzetti, L. 2000, MNRAS, 312, L9

Maeder, A. 1992, A\&A, 264, 105

Maraston, C. 1998, MNRAS, 300, 872

Marigo, P., Girardi, L., \& Bressan, A. 1999, A\&A, 344, 123

Marigo, P. 2001, A\&A, 370, 194

Portinari, L., Chiosi, C., \& Bressan, A. 1998, A\&A, 334, 505

Renzini, A., \& Buzzoni, A. 1986, in Spectral Evolution of Galaxies, ed. C. Chiosi, \& A. Renzini (Dordrecht: Reidel), 195

Renzini, A., \& Voli, M. 1981, A\&A, 94, 175

Renzini, A. 1998, AJ, 115, 2459

Tinsley, B. M. 1980, Fund. Cosmic Phys., 5, 287

Tuchman, Y., Glasner, A., \& Barkat, Z. 1983, ApJ, 268, 356

van den Hoek, L. B., \& Groenewegen, M. A. T. 1997, A\&AS, 123,305

Vazdekis, A., Casuso, E., Peletier, R. F., \& Beckman, J. E. 1996, ApJS, 106, 307

Woosley, S. E., \& Weaver, T. A. 1995, ApJS, 101, 181 